Article

\title{
On Some New Neighborhood Degree-Based Indices for Some Oxide and Silicate Networks
}

\author{
Sourav Mondal ${ }^{1}\left(\mathbb{D}\right.$, Nilanjan De ${ }^{2, *(1)}$ and Anita Pal ${ }^{1(\mathbb{C}}$ \\ 1 Department of Mathematics, National Institute of Technology Durgapur, Durgapur-713209, India \\ 2 Department of Basic Sciences and Humanities (Mathematics), Calcutta Institute of Engineering and \\ Management, Kolkata-700040, India \\ * Correspondence: de.nilanjan@rediffmail.com; Tel.: +91-98-3127-8235
}

Received: 16 June 2019; Accepted: 21 August 2019; Published: 24 August 2019

\begin{abstract}
Topological indices are numeric quantities that describes the topology of molecular structure in mathematical chemistry. An important area of applied mathematics is the chemical reaction network theory. Real-world problems can be modeled using this theory. Due to its worldwide applications, chemical networks have attracted researchers since their foundation. In this report, some silicate and oxide networks are studied, and exact expressions of some newly-developed neighborhood degree-based topological indices named as the neighborhood Zagreb index $\left(M_{N}\right)$, the neighborhood version of the forgotten topological index $\left(F_{N}\right)$, the modified neighborhood version of the forgotten topological index $\left(F_{N}^{*}\right)$, the neighborhood version of the second Zagreb index $\left(M_{2}^{*}\right)$, and neighborhood version of the hyper Zagreb index $\left(H M_{N}\right)$ are obtained for the aforementioned networks. In addition, a comparison among all the indices is shown graphically.
\end{abstract}

Keywords: topological indices; silicate and oxide networks; copper (II) oxide network

\section{Introduction}

Networks connect nodes that are somehow interconnected. Numerous personal computers connected together form a network. Cell phone users create a network. The networking process involves examining the best way to implement a network. Cheminformatics is a new area of research in which chemistry, mathematics, and information science are combined. That is how it caught the attention of researchers around the world. In this paper, we consider different silicate and oxide networks. The most abundant minerals in the Earth's crust are silicates. In most frequently-found silicates, including almost all silicate minerals found in the crust of the Earth, each silicon atom comprises the center of a tetrahedron (Figure 1), the corners of which are occupied by oxygen atoms, connected to it by single covalent bonds according to the octet rule. Silicates are obtained by fusing metal oxides or metal carbonates from sand. We can get various silicate structures by combining different tetrahedron silicates. Similarly, silicate networks are built by different silicate structures. The oxide networks are obtained by removing silicon atoms from the center of tetrahedra. Here, we also consider the copper (II) oxide (cupric oxide) network. Copper has enormous applications in medical science. It plays an important role in the synthesis and stabilization of skin proteins, and it also has potent biocidal properties [1]. The copper (II) oxide (cupric oxide) forms an inorganic chemical compound $\mathrm{C} u O$. This mineral is important in animals and plants. Copper (II) oxide is utilized in mineral and vitamin supplements as a copper source and is regarded as safe. 


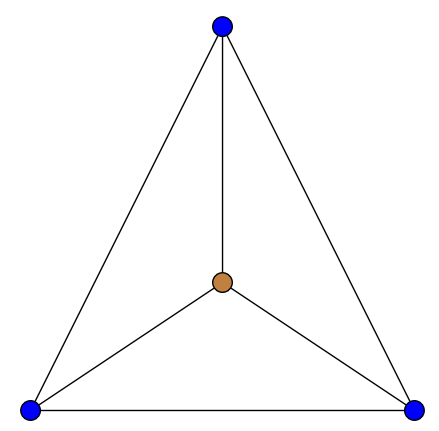

Figure 1. Silicate tetrahedra $\left(\mathrm{SiO}_{4}\right)$.

Molecular graphs are pictorial models of chemical compounds, assigning atoms as nodes and chemical bonds as edges. Throughout this report, we consider only molecular graphs [2-4]. We consider $V(G)$ and $E(G)$ as vertex and edge sets, respectively, for a graph $G$. The degree of a vertex $u \in V(G)$, denoted by $d_{G}(u)$, is the number of edges that are incident to $u$ in $G$. We say that a node $u$ is a neighbor of another node $v$ if $u$ is adjacent to $v$ in $G$. Here, $\delta_{G}(v)$ represents the totality of degrees of all neighbors of $v$ in $G$, i.e.,

$$
\delta_{G}(v)=\sum_{u \in N_{G}(v)} d_{G}(u)
$$

where $N_{G}(v)=\{u \in V(G): u v \in E(G)\}$. Graph theory creates a link between mathematics and chemistry by a useful tool named the topological index. A topological index is a real number associated with a graph obtained by certain rules such that two isomorphic graphs have the same topological index. It characterizes the topology of molecular structure. Topological indices play a vital role in the quantitative structure-property relationship (QSPR) and the quantitative structure-activity relationship (QSAR) models to predict different physico-chemical properties and bioactivity that help in drug discovery. Its applications in various fields such as nano-science, biotechnology, etc., are also remarkable. That is why it has attracted researchers' attention worldwide. The journey of topological indices started when chemist Harold Wiener introduced the Wiener index [5] in 1947. Several topological indices have been introduced over the past decades based on the vertex degree. Inspired by the works [6-8], we designed some new degree-based topological indices $[9,10]$ having nice correlations with entropy and the acentric factor. Their mean isomer degeneracy is also remarkable. These indices are defined as follows.

The neighborhood Zagreb index is denoted by $M_{N}(G)$ and is defined as:

$$
M_{N}(G)=\sum_{v \in V(G)} \delta_{G}(v)^{2} .
$$

The neighborhood version of the forgotten topological index is denoted by $F_{N}(G)$ and is defined as:

$$
F_{N}(G)=\sum_{v \in V(G)} \delta_{G}(v)^{3}
$$

The modified neighborhood version of the forgotten topological index is denoted by $F_{N}^{*}(G)$ and is defined as:

$$
F_{N}^{*}(G)=\sum_{u v \in E(G)}\left[\delta_{G}(u)^{2}+\delta_{G}(v)^{2}\right]
$$


The neighborhood version of the second Zagreb index is denoted by $M_{2}^{*}(G)$ and is defined by:

$$
M_{2}^{*}(G)=\sum_{u v \in E(G)}\left[\delta_{G}(u) \delta_{G}(v)\right]
$$

The neighborhood version of the hyper Zagreb index is denoted by $H M_{N}(G)$ and is defined by:

$$
H M_{N}(G)=\sum_{u v \in E(G)}\left[\delta_{G}(u)+\delta_{G}(v)\right]^{2} .
$$

In [11-19], topological indices for different chemically important graphs were discussed. Baig et al. [20] derived topological indices for some silicate and oxide networks. Javaid et al. [21] discussed the topological properties of rhombus-type silicate and oxide networks. In [22], topological indices for the copper (II) oxide network were obtained. For further work related to this field, readers are referred to [23-26]. Motivated by those works, in this paper, we obtain some exact expressions of five novel indices described above for some silicate and oxide networks and compare the results graphically.

This paper is organized as follows. In the next section, the motivation behind this work is described. The usefulness of these indices is illustrated here. The computation of the topological indices for different silicate and oxide networks using vertex and edge partition procedures is presented in Section 3. The graphical representation of the results is shown here using MATLAB (9.2, The MathWorks, Inc., Natick, MA, USA) and Maple (2015.1, Maplesoft, Waterloo, ON Canada) software. We conclude this report with a comparative study of the indices for different networks under consideration.

\section{Motivation}

In order to determine the usefulness of a topological index to predict the physico-chemical behavior of a chemical compound, the correlation coefficient $(r)$ between the physico-chemical properties and topological indices is calculated. In QSPR/QSAR analysis, the topological indices for which $r^{2}$ exceeds 0.8 are very useful. In $[9,10]$, it was shown that the $r^{2}$ values of five topological indices $M_{N}, F_{N}, F_{N}^{*}, M_{2}^{*}$, and $H M_{N}$ have excellent correlations with entropy and the acentric factor for octane isomers. The $r^{2}$ values of those indices with entropy are $0.989,0.88,0.868,0.926$, and 0.879 , respectively. The $r^{2}$ values of those indices with the acentric factor are $0.907,0.989,0.952,0.819$, and 0.961 , respectively. Linear correlations of these indices with entropy and the acentric factor are depicted in Figure 2. The above-mentioned indices are therefore effective in QSPR/QSAR analysis with high statistical accuracy. Furthermore, their mean isomer degeneracy is also remarkable in comparison with other degree-based topological indices [9,10],

which provides assurance of their good isomer discrimination ability. Calculating these indices for distinct chemically-important networks and composite graphs is therefore worthwhile. Here, we consider some oxide and silicate networks. 

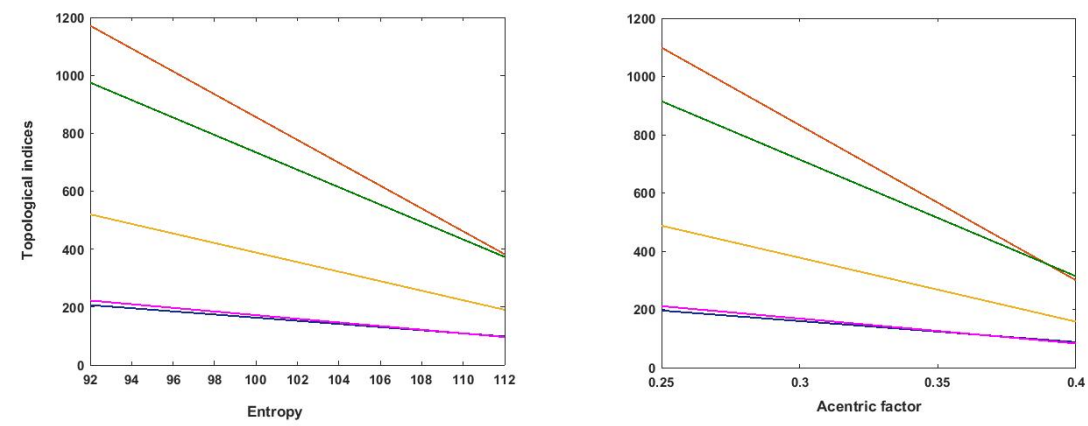

$-\mathrm{M}_{\mathrm{N}}-\mathrm{F}_{\mathrm{N}}-\mathrm{F}_{\mathrm{N}}{ }^{*}-\mathrm{M}_{2}{ }^{*}-\mathrm{HM}_{\mathrm{N}}$

Figure 2. Linear fitting of five topological indices with entropy and the acentric factor.

\section{Computation of Topological Indices}

In this section, we first discuss the vertex and edge partition of the networks, and then, using that partition, we obtain five topological indices discussed in the previous section for the networks. Here, we consider dominating silicate $(D S L(n))$ and oxide $(D O X(n)))$ networks, regular triangulene silicate $(\operatorname{RTSL}(n))$ and oxide $(\operatorname{RTOX}(n))$ networks, rhombus silicate $(R H S L(n))$ and oxide $(\operatorname{RHOX}(n))$ networks, and the copper (II) oxide $(\mathrm{CuO})$ network. The construction procedures of these networks are discussed in detail in $[21,22,26]$. The first three types of networks can be constructed from a honeycomb network $H C(n)$ of dimension $n$ (number of hexagonal layers) [26]. The structure of $H C(3)$ is shown in Figure 3. To create the vertex and edge partitions, we consider the following notations.

$$
\begin{gathered}
V_{i}=\left\{u \in V(G): \delta_{G}(u)=i\right\}, \\
E_{(p, q)}=\left\{u v \in E(G): \delta_{G}(u)=p, \delta_{G}(v)=q\right\} .
\end{gathered}
$$

By $\left|V_{i}\right|$ and $\left|E_{(p, q)}\right|$, we mean the cardinality of the sets $V_{i}$ and $E_{(p, q)}$, respectively.

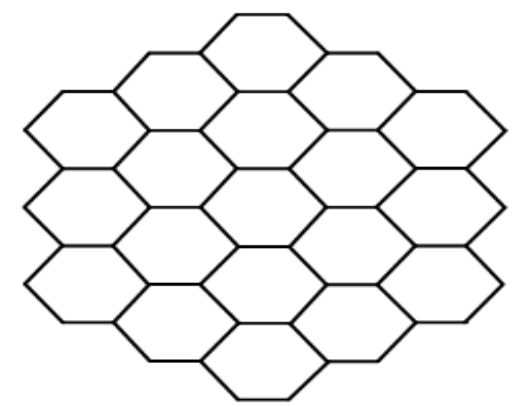

Figure 3. Honeycomb network $H C(3)$ of dimension three.

\subsection{Dominating Silicate $(\operatorname{DSL}(n))$ and Oxide $(\operatorname{DOX}(n)))$ Networks}

In this subsection, we obtain the five aforementioned indices for dominating silicate $(D S L(n))$ and oxide $(\operatorname{DOX}(n))$ networks. The structures of $\operatorname{DSL}(3)$ and $\operatorname{DOX}(3)$ are depicted in Figures 4 and 5, respectively. Dominating silicate networks have $45 n^{2}-39 n+12$ nodes and $108 n^{2}-108 n+36$ edges [20,26]. 
Dominating oxide networks admit $27 n^{2}-21 n+6$ nodes and $54 n^{2}-54 n+18$ edges [20,26]. Vertex and edge partitions for dominating silicate networks are as follows.

$$
\begin{gathered}
V(D S L(n))=V_{15} \cup V_{18} \cup V_{24} \cup V_{27} \cup V_{30}, \\
E(D S L(n))=E_{(15,15)} \cup E_{(15,24)} \cup E_{(15,27)} \cup E_{(18,27)} \cup E_{(18,30)} \cup E_{(24,24)} \\
\cup E_{(24,27)} \cup E_{(27,30)} \cup E_{(30,30)}
\end{gathered}
$$

Vertex and edge partitions for dominating oxide networks are as follows.

$$
\begin{gathered}
V(\operatorname{DOX}(n))=V_{8} \cup V_{12} \cup V_{14} \cup V_{16}, \\
E(\operatorname{DOX}(n))=E_{(8,12)} \cup E_{(8,14)} \cup E_{(12,12)} \cup E_{(12,14)} \cup E_{(14,16)} \cup E_{(16,16)} .
\end{gathered}
$$

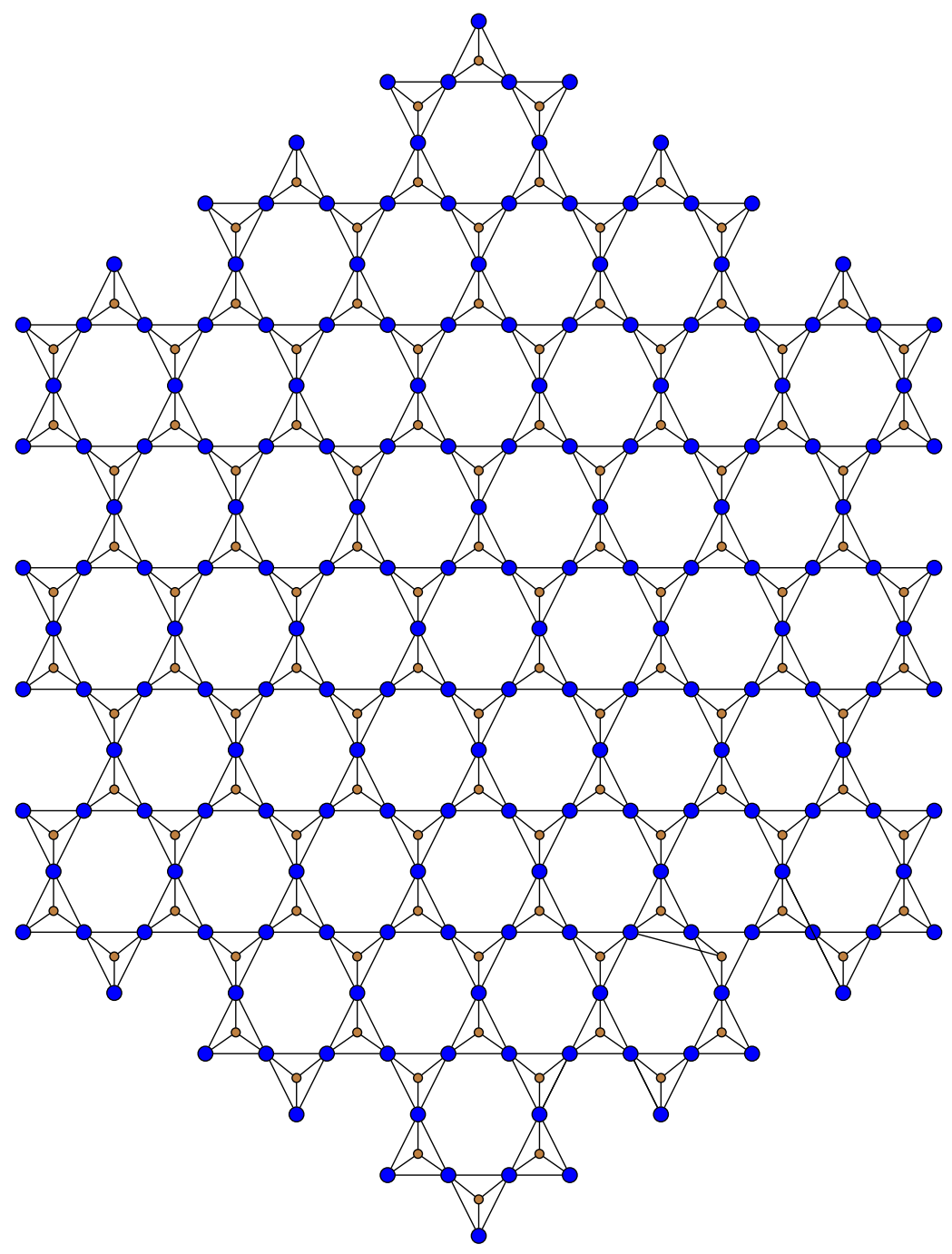

Figure 4. Dominating silicate network (DSL(3)). 


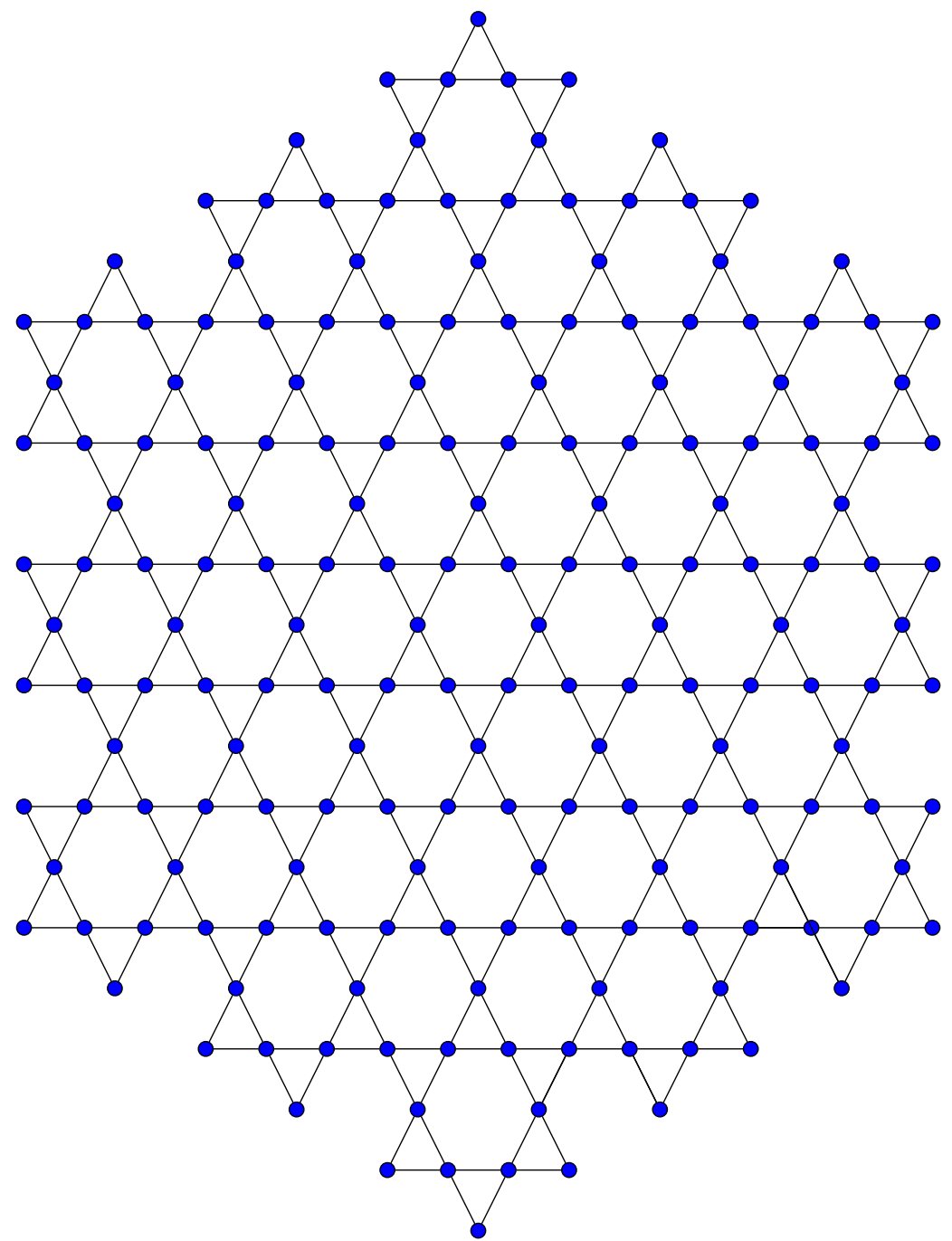

Figure 5. Dominating oxide network $(\operatorname{DOX}(3))$.

Theorem 1. The neighborhood Zagreb index $\left(M_{N}\right)$ of the dominating silicate $(\operatorname{DSL}(n))$ and oxide $\left.(\operatorname{DOX}(n))\right)$ networks for $n \geq 2$ is given by:

(i) $\quad M_{N}(D S L(n))=30,132 n^{2}-38,016 n+14,040$,

(ii) $M_{N}(D O X(n))=6912 n^{2}-9072 n+3408$.

Proof. The general formula of the neighborhood Zagreb index is given by:

$$
M_{N}(G)=\sum_{v \in V(G)} \delta_{G}(v)^{2}
$$

(i) Let $G \cong D S L(n)$. Now, applying vertex partition (1) on the definition of the neighborhood Zagreb index, we get:

$$
M_{N}(G)=\sum_{v \in V_{15}} \delta_{G}(v)^{2}+\sum_{v \in V_{18}} \delta_{G}(v)^{2}+\sum_{v \in V_{24}} \delta_{G}(v)^{2}+\sum_{v \in V_{27}} \delta_{G}(v)^{2}+\sum_{v \in V_{30}} \delta_{G}(v)^{2}
$$


Using Table 1, we obtain:

$$
\begin{aligned}
M_{N}(G)= & \left|V_{15}\right|\left(15^{2}\right)+\left|V_{18}\right|\left(18^{2}\right)+\left|V_{24}\right|\left(24^{2}\right)+\left|V_{27}\right|\left(27^{2}\right)+\left|V_{30}\right|\left(30^{2}\right) \\
= & (24 n-12)\left(15^{2}\right)+\left(18 n^{2}-30 n+12\right)\left(18^{2}\right)+(6 n)\left(24^{2}\right) \\
& +12(n-1)\left(27^{2}\right)+\left(27 n^{2}-51 n+24\right)\left(30^{2}\right) \\
= & 30,132 n^{2}-38,016 n+14,040 .
\end{aligned}
$$

Table 1. Vertex partition of dominating silicate networks $(D S L(n))$ with dimension $n$.

\begin{tabular}{cccccc}
\hline$V_{\boldsymbol{i}}$ & $V_{\mathbf{1 5}}$ & $V_{\mathbf{1 8}}$ & $V_{\mathbf{2 4}}$ & $V_{\mathbf{2 7}}$ & $V_{\mathbf{3 0}}$ \\
\hline$\left|V_{i}\right|$ & $24 n-12$ & $18 n^{2}-30 n+12$ & $6 n$ & $12(n-1)$ & $27 n^{2}-51 n+24$ \\
\hline
\end{tabular}

(ii) Let $G \cong \operatorname{DOX}(n)$. Now, using vertex partition (3) and Table 2 on the definition of the neighborhood Zagreb index, we obtain:

$$
\begin{aligned}
M_{N}(G)= & \left|V_{8}\right|\left(8^{2}\right)+\left|V_{12}\right|\left(12^{2}\right)+\left|V_{14}\right|\left(14^{2}\right)+\left|V_{16}\right|\left(16^{2}\right) \\
= & (12 n-6)\left(8^{2}\right)+(6 n)\left(12^{2}\right)+12(n-1)\left(14^{2}\right) \\
& +\left(27 n^{2}-51 n+24\right)\left(16^{2}\right) .
\end{aligned}
$$

Table 2. Vertex partition of dominating oxide networks $(D O X(n))$ with dimension $n$.

\begin{tabular}{ccccc}
\hline$V_{i}$ & $V_{\mathbf{8}}$ & $V_{\mathbf{1 2}}$ & $V_{\mathbf{1 4}}$ & $V_{\mathbf{1 6}}$ \\
\hline$\left|V_{i}\right|$ & $12 n-6$ & $6 n$ & $12(n-1)$ & $27 n^{2}-51 n+24$ \\
\hline
\end{tabular}

After simplifying and arranging the terms, the required result can be obtained easily.

Hence the proof.

Theorem 2. The neighborhood versions of the forgotten topological index $\left(F_{N}\right)$ of the dominating silicate $(D S L(n))$ and oxide $(\operatorname{DOX}(n)))$ networks for $n \geq 2$ are given by:

(i) $F_{N}(D S L(n))=833,976 n^{2}-1,151,820 n+441,288$,

(ii) $F_{N}(\operatorname{DOX}(n))=110,592 n^{2}-159,456 n+62,304$.

Proof. The general formulation of the neighborhood version of the forgotten topological index is given by:

$$
F_{N}(G)=\sum_{v \in V(G)} \delta_{G}(v)^{3}
$$

(i) Let $G \cong D S L(n)$. Using vertex partition (1) and Table 1 on the above formula, we have:

$$
\begin{aligned}
F_{N}(G)= & (24 n-12)\left(15^{3}\right)+\left(18 n^{2}-30 n+12\right)\left(18^{3}\right)+(6 n)\left(24^{3}\right)+ \\
& 12(n-1)\left(27^{3}\right)+\left(27 n^{2}-51 n+24\right)\left(30^{3}\right) \\
= & 833,976 n^{2}-1,151,820 n+441,288 .
\end{aligned}
$$


(ii) Let $G \cong \operatorname{DOX}(n)$. Using vertex partition (3) and Table 2, the general formula of the $F_{N}$ index gives the following computation.

$$
\begin{aligned}
F_{N}(G)= & (12 n-6)\left(8^{3}\right)+(6 n)\left(12^{3}\right)+12(n-1)\left(14^{3}\right)+ \\
& +\left(27 n^{2}-51 n+24\right)\left(16^{3}\right) \\
= & 110,592 n^{2}-159,456 n+62,304 .
\end{aligned}
$$

Hence the proof.

Theorem 3. The modified neighborhood versions of the forgotten topological index $\left(F_{N}^{*}\right)$ of the dominating silicate $(\operatorname{DSL}(n))$ and oxide $(\operatorname{DOX}(n)))$ networks for $n \geq 2$ are given by:

(i) $F_{N}^{*}(D S L(n))=163,296 n^{2}-215,136 n+80,676$,

(ii) $F_{N}^{*}(D O X(n))=27,648 n^{2}-37,824 n+14,400$.

Proof. The general formula of the modified neighborhood version of the forgotten topological index is given by:

$$
F_{N}^{*}(G)=\sum_{u v \in E(G)}\left[\delta_{G}(u)^{2}+\delta_{G}(v)^{2}\right]
$$

(i) Let $G$ be the dominating silicate network with dimension $n$. Then, applying edge partition (2) on the definition of the $F_{N}^{*}$ index, we have:

$$
\begin{aligned}
F_{N}^{*}(G)= & \sum_{u v \in E(G)}\left[\delta_{G}(u)^{2}+\delta_{G}(v)^{2}\right] \\
= & \sum_{u v \in E_{(15,15)}}\left[\delta_{G}(u)^{2}+\delta_{G}(v)^{2}\right]+\sum_{u v \in E_{(15,24)}}\left[\delta_{G}(u)^{2}+\delta_{G}(v)^{2}\right] \\
& +\sum_{u v \in E_{(15,27)}}\left[\delta_{G}(u)^{2}+\delta_{G}(v)^{2}\right]+\sum_{u v \in E_{(18,27)}}\left[\delta_{G}(u)^{2}+\delta_{G}(v)^{2}\right] \\
& +\sum_{u v \in E_{(18,30)}}\left[\delta_{G}(u)^{2}+\delta_{G}(v)^{2}\right]+\sum_{u v \in E_{(24,24)}}\left[\delta_{G}(u)^{2}+\delta_{G}(v)^{2}\right] \\
& +\sum_{u v \in E_{(24,27)}}\left[\delta_{G}(u)^{2}+\delta_{G}(v)^{2}\right]+\sum_{u v \in E_{(27,30)}}\left[\delta_{G}(u)^{2}+\delta_{G}(v)^{2}\right] \\
& +\sum_{u v \in E_{(30,30)}}\left[\delta_{G}(u)^{2}+\delta_{G}(v)^{2} .\right.
\end{aligned}
$$

Using Table 3, we can obtain:

$$
\begin{aligned}
F_{N}^{*}(G)= & \left|E_{(15,15)}\right|\left(15^{2}+15^{2}\right)+\left|E_{(15,24)}\right|\left(15^{2}+24^{2}\right)+\left|E_{(15,27)}\right|\left(15^{2}+27^{2}\right) \\
& +\left|E_{(18,27)}\right|\left(18^{2}+27^{2}\right)+\left|E_{(18,30)}\right|\left(18^{2}+30^{2}\right)+\left|E_{(24,24)}\right|\left(24^{2}+24^{2}\right) \\
& +\left|E_{(24,27)}\right|\left(24^{2}+27^{2}\right)+\left|E_{(27,30)}\right|\left(27^{2}+30^{2}\right)+\left|E_{(30,30)}\right|\left(30^{2}+30^{2}\right) \\
= & 6(2 n-1)\left(15^{2}+15^{2}\right)+(24 n)\left(15^{2}+24^{2}\right)+24(n-1)\left(15^{2}+27^{2}\right) \\
& +12(n-1) \mid\left(18^{2}+27^{2}\right)+\left(54 n^{2}-102 n+48\right)\left(18^{2}+30^{2}\right) \\
& +(6)\left(24^{2}+24^{2}\right)+12(n-1)\left(24^{2}+27^{2}\right)+24(n-1)\left(27^{2}+30^{2}\right) \\
& +\left(54 n^{2}-114 n+60\right)\left(30^{2}+30^{2}\right) .
\end{aligned}
$$


Table 3. Edge partition of dominating silicate networks $(D S L(n))$ with dimension $n$.

\begin{tabular}{cccccccccc}
\hline$E_{(p, q)}$ & $E_{(15,15)}$ & $E_{(15,24)}$ & $E_{(15,27)}$ & $E_{(18,27)}$ & $E_{(18,30)}$ & $E_{(24,24)}$ & $E_{(24,27)}$ & $E_{(27,30)}$ & $E_{(30,30)}$ \\
\hline$\left|E_{(p, q)}\right|$ & $6(2 n-1)$ & $24 n$ & $24(n-1)$ & $12(n-1)$ & $54 n^{2}-102 n+48$ & 6 & $12(n-1)$ & $24(n-1)$ & $54 n^{2}-114 n+60$ \\
\hline
\end{tabular}

After simplification, the desired result is clear.

(ii) Let $G$ be the dominating silicate network with dimension $n$.

Now, applying the vertex partition (4) and Table 4 on the definition of the $F_{N}^{*}$ index, we have:

$$
\begin{aligned}
F_{N}^{*}(G)= & \left|E_{(8,12)}\right|\left(8^{2}+12^{2}\right)+\left|E_{(8,14)}\right|\left(8^{2}+14^{2}\right)+\left|E_{(12,12)}\right|\left(12^{2}+12^{2}\right) \\
& +\left|E_{(12,14)}\right|\left(12^{2}+14^{2}\right)+\left|E_{(14,16)}\right|\left(14^{2}+16^{2}\right)+\left|E_{(16,16)}\right|\left(16^{2}+16^{2}\right) \\
= & (12 n)\left(8^{2}+12^{2}\right)+12(n-1)\left(8^{2}+14^{2}\right)+(6)\left(12^{2}+12^{2}\right) \\
& +12(n-1)\left(12^{2}+14^{2}\right)+24(n-1)\left(14^{2}+16^{2}\right) \\
& +\left(54 n^{2}-114 n+60\right)\left(16^{2}+16^{2}\right) \\
= & 27,648 n^{2}-37,824 n+14,400 .
\end{aligned}
$$

Table 4. Edge partition of dominating oxide networks $(\operatorname{DOX}(n))$ with dimension $n$.

\begin{tabular}{ccccccc}
\hline$E_{(p, q)}$ & $E_{(8,12)}$ & $E_{(8,14)}$ & $E_{(12,12)}$ & $E_{(12,14)}$ & $E_{(14,16)}$ & $E_{(16,16)}$ \\
\hline$\left|E_{(p, q)}\right|$ & $12 n$ & $12(n-1)$ & 6 & $12(n-1)$ & $24(n-1)$ & $54 n^{2}-114 n+60$ \\
\hline
\end{tabular}

Hence the theorem.

Theorem 4. The neighborhood versions of the second Zagreb index $\left(M_{2}^{*}\right)$ of the dominating silicate $(D S L(n))$ and oxide $(D O X(n)))$ networks for $n \geq 2$ are given by:

(i) $\quad M_{2}^{*}(\operatorname{DSL}(n))=77,760 n^{2}-103,572 n+39,258$,

(ii) $M_{2}^{*}(\operatorname{DOX}(n))=13,824 n^{2}-19,296 n+7488$.

Proof. The general formula of the neighborhood version of the second Zagreb index is given by:

$$
M_{2}^{*}(G)=\sum_{u v \in E(G)}\left[\delta_{G}(u) \delta_{G}(v)\right]
$$

(i) Let $G \cong D S L(n)$. Now, applying the edge partition (2) and Table 3 on the definition of the neighborhood version of the second Zagreb index, we get the desired result as follows.

$$
\begin{aligned}
M_{2}^{*}(G)= & 6(2 n-1)(15.15)+24 n(15.24)+24(n-1)(15.27)+12(n-1)(18.27) \\
& +\left(54 n^{2}-102 n+48\right)(18.30)+6(24.24)+12(n-1)(24.27) \\
& +24(n-1)(27.30)+\left(54 n^{2}-114 n+60\right)(30.30) \\
= & 77,760 n^{2}-103,572 n+39,258 .
\end{aligned}
$$


(ii) Let $G \cong D O X(n)$. Applying the edge partition (4) and Table 4 on the definition of the neighborhood version of the second Zagreb index, we get the desired result as follows.

$$
\begin{aligned}
M_{2}^{*}(G)= & 12 n(8.12)+12(n-1)(8.14)+6(12.12)+12(n-1)(12 \\
& +24(n-1)(14.16)+\left(54 n^{2}-114 n+60\right)(16.16) \\
= & 13,824 n^{2}-19,296 n+7488
\end{aligned}
$$

Hence the proof.

Theorem 5. The neighborhood versions of the hyper Zagreb index $\left(H M_{N}\right)$ of the dominating silicate $(D S L(n))$ and oxide $(\operatorname{DOX}(n)))$ networks for $n \geq 2$ are given by:

(i) $H_{N}(D S L(n))=318,816 n^{2}-422,280 n+159,192$,

(ii) $H_{N}(\operatorname{DOX}(n))=55,296 n^{2}-76,416 n+29,376$.

Proof. (i) Let $G \cong D S L(n)$. Now, applying edge partition (2) and Table 3 on the definition of the $H M_{N}$ index, we get the following computation.

$$
\begin{aligned}
H M_{N}(G)= & \sum_{u v \in E(G)}\left[\delta_{G}(u)+\delta_{G}(v)\right]^{2} \\
= & 6(2 n-1)(15+15)^{2}+(24 n)(15+24)^{2}+24(n-1)(15+27)^{2} \\
& +12(n-1)\left((18+27)^{2}+\left(54 n^{2}-102 n+48\right)(18+30)^{2}\right. \\
& +6(24+24)^{2}+12(n-1)(24+27)^{2}+24(n-1)(27+30)^{2} \\
& +\left(54 n^{2}-114 n+60\right)(30+30)^{2} \\
= & 318,816 n^{2}-422,280 n+159,192 .
\end{aligned}
$$

(ii) Let $G \cong \operatorname{DOX}(n)$. Now, applying edge partition (4) and Table 4 on the definition of the $H M_{N}$ index, we get the following computation.

$$
\begin{aligned}
H M_{N}(G)= & \sum_{u v \in E(G)}\left[\delta_{G}(u)+\delta_{G}(v)\right]^{2} \\
= & (12 n)(8+12)^{2}+12(n-1)(8+14)^{2}+6(12+12)^{2} \\
& +12(n-1)\left((12+14)^{2}+24(n-1)(14+16)^{2}\right. \\
& +\left(54 n^{2}-114 n+60\right)(16+16)^{2} \\
= & 55,296 n^{2}-76,416 n+29,376 .
\end{aligned}
$$

Hence the theorem.

Five topological indices for $\operatorname{DSL}(n)$ and $\operatorname{DOX}(n)$ are shown graphically in Figure 6. 


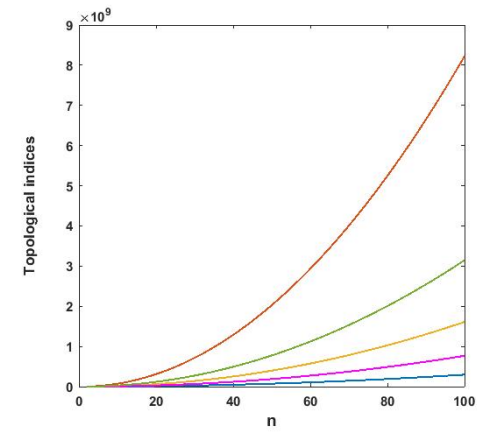

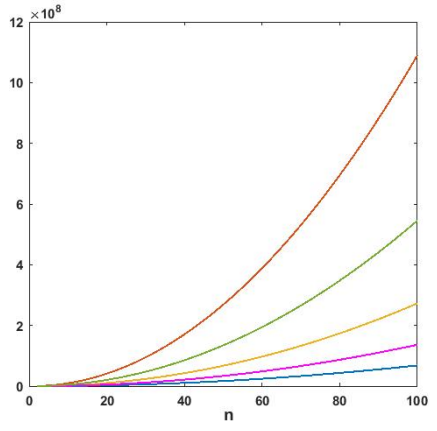

(b)

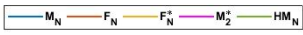

Figure 6. Different topological indices for dominating (a) silicate and (b) oxide networks.

\subsection{Regular Triangulene Silicate (RTSL $(n))$ and Oxide $(R T O X(n))$ Networks}

In this subsection, we obtain the five aforementioned indices for regular triangulene silicate $(R T S L(n))$ and oxide $(\operatorname{RTOX}(n))$ networks. The structures of $\operatorname{RTSL}(5)$ and $\operatorname{RTOX}(5)$ are shown in Figures 7 and 8, respectively. Using the method described in [26], it can be said that regular triangulene silicate networks have $\frac{5 n^{2}+15 n+2}{2}$ nodes and $3 n^{2}+31 n-28$ edges. The regular triangulene oxide network admits $\frac{3 n^{2}+9 n+2}{2}$ nodes and $3 n^{2}+6 n$ edges $[23,26]$. Vertex and edge partitions for the regular triangulene silicate network are as follows.

$$
\begin{gathered}
V(R T S L(n))=V_{12} \cup V_{15} \cup V_{18} \cup V_{24} \cup V_{27} \cup V_{30}, \\
E(R T S L(n))=E_{(12,12)} \cup E_{(12,15)} \cup E_{(12,24)} \cup E_{(15,15)} \cup E_{(15,24)} \cup E_{(15,27)} \cup E_{(18,24)} \\
\cup E_{(18,27)} \cup E_{(18,30)} \cup E_{(24,24)} \cup E_{(24,27)} \cup E_{(27,27)} \cup E_{(27,30)} \cup E_{(30,30) .} .
\end{gathered}
$$

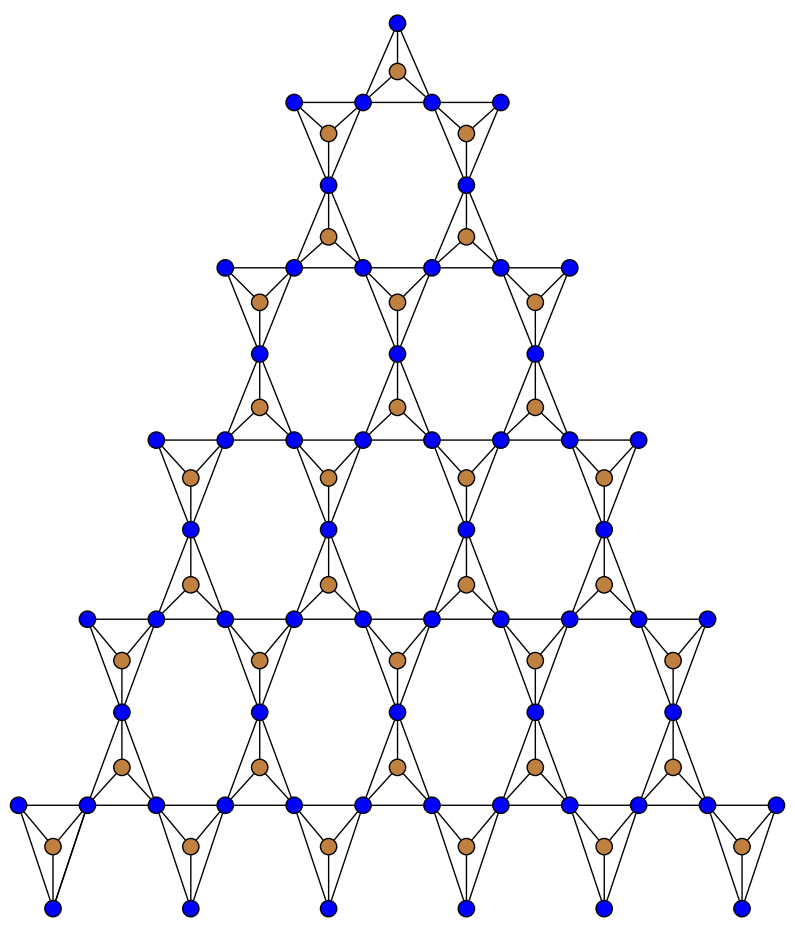

Figure 7. Regular triangulene silicate network (RTSL(5)). 


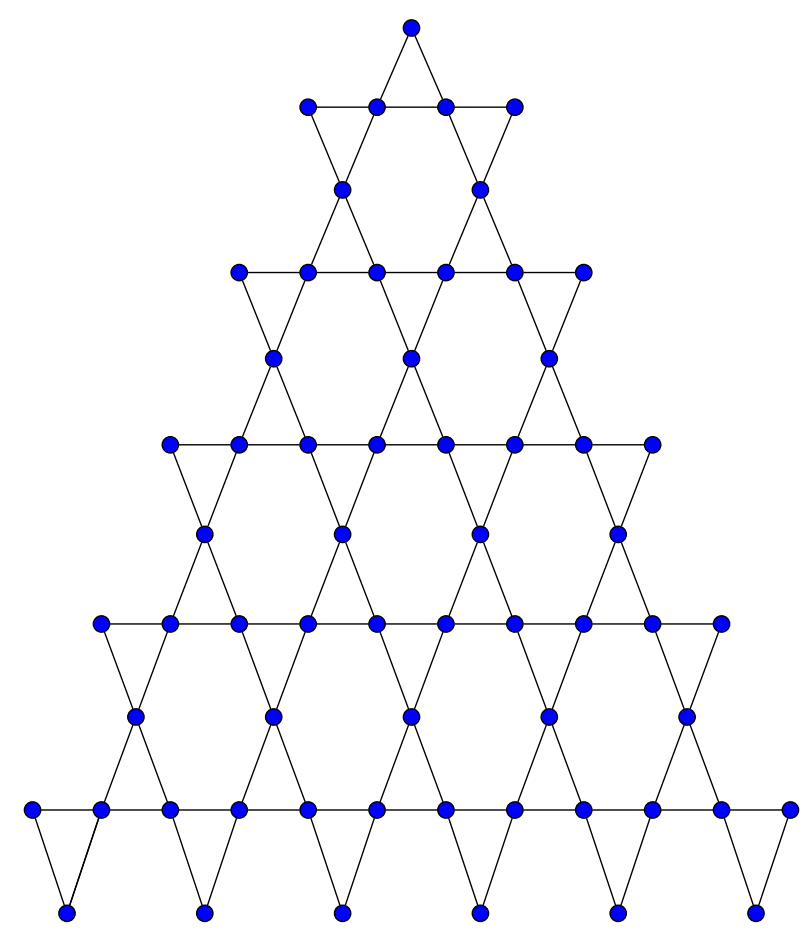

Figure 8. Regular triangulene oxide network $(R T O X(5))$.

Vertex and edge partitions for the regular triangulene oxide network are as follows.

$$
\begin{gathered}
V(\operatorname{RTOX}(n))=V_{6} \cup V_{8} \cup V_{12} \cup V_{14} \cup V_{16}, \\
E(R T O X(n))=E_{(6,6)} \cup E_{(6,12)} \cup E_{(8,12)} \cup E_{(8,14)} \cup E_{(12,12)} \cup E_{(12,14)} \cup E_{(14,14)} \cup E_{(14,16)} \cup E_{(16,16)} .
\end{gathered}
$$

Theorem 6. The neighborhood Zagreb index $\left(M_{N}\right)$ of the regular triangulene silicate $(R T S L(n))$ and oxide $(\operatorname{RTOX}(n)))$ networks for $n \geq 2$ is given by:

(i) $\quad M_{N}(\operatorname{RTSL}(n))=1674 n^{2}+1674 n-702$,

(ii) $M_{N}(R T O X(n))=384 n^{2}+216 n-208$.

Proof. The general formula of the neighborhood Zagreb index is given by:

$$
M_{N}(G)=\sum_{v \in V(G)} \delta_{G}(v)^{2} .
$$

(i) Let $G \cong \operatorname{RTSL}(n)$. Now, apply vertex partition (5) and Table 5 on the definition of the neighborhood Zagreb index, we get:

$$
\begin{aligned}
M_{N}(G)= & \left|V_{12}\right|\left(12^{2}\right)+\left|V_{15}\right|\left(15^{2}\right)+\left|V_{18}\right|\left(18^{2}\right)+\left|V_{24}\right|\left(24^{2}\right) \\
& +\left|V_{27}\right|\left(27^{2}\right)+\left|V_{30}\right|\left(30^{2}\right) \\
= & (4)\left(12^{2}\right)+(6 n-2)\left(15^{2}\right)+\left(n^{2}\right)\left(18^{2}\right)+(4)\left(24^{2}\right)+(6 n-8)\left(27^{2}\right) \\
& +\left(\frac{3 n^{2}-9 n+6}{2}\right)\left(30^{2}\right) .
\end{aligned}
$$


Table 5. Vertex partition of regular triangulene silicate networks $(\operatorname{RTSL}(n))$ with dimension $n$.

\begin{tabular}{ccccccc}
\hline$V_{\boldsymbol{i}}$ & $V_{\mathbf{1 2}}$ & $V_{\mathbf{1 5}}$ & $V_{\mathbf{1 8}}$ & $V_{\mathbf{2 4}}$ & $V_{\mathbf{2 7}}$ & $V_{\mathbf{3 0}}$ \\
\hline$\left|V_{i}\right|$ & 4 & $6 n-2$ & $n^{2}$ & 4 & $6 n-8$ & $\frac{3 n^{2}-9 n+6}{2}$ \\
\hline
\end{tabular}

After simplification, the desired result can be obtained easily.

(ii) Let $G \cong R T O X(n)$. Now, using vertex partition (7) and Table 6 on the definition of the neighborhood Zagreb index, we obtain:

$$
\begin{aligned}
M_{N}(G)= & \left|V_{6}\right|\left(6^{2}\right)+\left|V_{8}\right|\left(8^{2}\right)+\left|V_{12}\right|\left(12^{2}\right)+\left|V_{14}\right|\left(14^{2}\right)+\left|V_{16}\right|\left(16^{2}\right) \\
= & (4)\left(6^{2}\right)+(3 n-2)\left(8^{2}\right)+(4)\left(12^{2}\right)+(6 n-8)\left(14^{2}\right) \\
& +\left(\frac{3 n^{2}-9 n+6}{2}\right)\left(16^{2}\right) .
\end{aligned}
$$

Table 6. Vertex partition of regular triangulene oxide networks $(R T O X(n))$ with dimension $n$.

\begin{tabular}{cccccc}
\hline$V_{i}$ & $V_{\mathbf{6}}$ & $V_{\mathbf{8}}$ & $V_{\mathbf{1 2}}$ & $V_{\mathbf{1 4}}$ & $V_{\mathbf{1 6}}$ \\
\hline$\left|V_{i}\right|$ & 4 & $3 n-2$ & 4 & $6 n-8$ & $\frac{3 n^{2}-9 n+6}{2}$ \\
\hline
\end{tabular}

After simplifying and arranging the terms, the required result can be obtained easily.

Hence the proof.

Theorem 7. The neighborhood version of the forgotten topological index $\left(F_{N}\right)$ of the regular triangulene silicate $(\operatorname{RTSL}(n))$ and oxide $(\operatorname{RTOX}(n)))$ networks for $n \geq 2$ is given by:

(i) $F_{N}(R T S L(n))=46,332 n^{2}+16,848 n+113,994$,

(ii) $\quad F_{N}(R T O X(n))=6144 n^{2}-432 n-2912$.

Proof. The general formulation of the neighborhood version of the forgotten topological index is given by:

$$
F_{N}(G)=\sum_{v \in V(G)} \delta_{G}(v)^{3}
$$

(i) Let $G$ be a regular triangulene silicate network having dimension $n$. Using vertex partition (5) and Table 5 on the above formula, we have:

$$
\begin{aligned}
F_{N}(G)= & (4)\left(12^{3}\right)+(6 n-2)\left(15^{3}\right)+\left(n^{2}\right)\left(18^{3}\right)+(4)\left(24^{3}\right)+ \\
& (6 n-8)\left(27^{3}\right)+\left(\frac{3 n^{2}-9 n+6}{2}\right)\left(30^{3}\right) \\
= & 46,332 n^{2}+16,848 n+113,994 .
\end{aligned}
$$


(ii) Let $G$ be a regular triangulene oxide network having dimension $n$. Using vertex partition (7) and Table 6 , the general formula of the $F_{N}$ index gives the following computation.

$$
\begin{aligned}
F_{N}(G)= & (4)\left(6^{3}\right)+(3 n-2)\left(8^{3}\right)+(4)\left(12^{3}\right)+(6 n-8)\left(14^{3}\right) \\
& +\left(\frac{3 n^{2}-9 n+6}{2}\right)\left(16^{3}\right) \\
= & 6144 n^{2}-432 n-2912 .
\end{aligned}
$$

Hence the proof.

Theorem 8. The modified neighborhood version of the forgotten topological index $\left(F_{N}^{*}\right)$ of the regular triangulene silicate $(\operatorname{RTSL}(n))$ and oxide $(\operatorname{RTOX}(n)))$ networks for $n \geq 2$ is given by:

(i) $F_{N}^{*}(\operatorname{RTSL}(n))=5400 n^{2}+28,278 n-38,394$,

(ii) $F_{N}^{*}(R T O X(n))=1536 n^{2}+480 n-864$.

Proof. The general formula of the modified neighborhood version of the forgotten topological index is given by:

$$
F_{N}^{*}(G)=\sum_{u v \in E(G)}\left[\delta_{G}(u)^{2}+\delta_{G}(v)^{2}\right]
$$

(i) Let $G \cong R T S L(n)$. Then, applying edge partition (6) and Table 7 on the definition of the $F_{N}^{*}$ index, we have:

$$
\begin{aligned}
F_{N}^{*}(G)= & (2)\left(12^{2}+12^{2}\right)+(4)\left(12^{2}+15^{2}\right)+(4)\left(12^{2}+24^{2}\right)+(3 n-2)\left(15^{2}\right. \\
& \left.+15^{2}\right)+(10)\left(15^{2}+24^{2}\right)+(12 n-16)\left(15^{2}+27^{2}\right)+(2)\left(18^{2}+\right. \\
& \left.24^{2}\right)+(6 n-8)\left(18^{2}+27^{2}\right)+(10 n-24)\left(18^{2}+30^{2}\right)+(1)\left(24^{2}\right. \\
& \left.+24^{2}\right)+(6)\left(24^{2}+27^{2}\right)+(6 n-7)\left(27^{2}+27^{2}\right)+(6 n-12)\left(27^{2}+\right. \\
& \left.30^{2}\right)+3(n-2)^{2}\left(30^{2}+30^{2}\right) .
\end{aligned}
$$

After simplification and arranging the terms, we can get the desired result.

(ii) Let $G \cong \operatorname{RTOX}(n)$. Now, applying the edge partition (8) and Table 8 on the definition of the $F_{N}^{*}$ index, we have:

$$
\begin{aligned}
F_{N}^{*}(G)= & \left|E_{(6,6)}\right|\left(6^{2}+6^{2}\right)+\left|E_{(6,12)}\right|\left(6^{2}+12^{2}\right)+\left|E_{(8,12)}\right|\left(8^{2}+12^{2}\right) \\
& +\left|E_{(8,14)}\right|\left(8^{2}+14^{2}\right)+\left|E_{(12,12)}\right|\left(12^{2}+12^{2}\right)+\left|E_{(12,14)}\right|\left(12^{2}+14^{2}\right) \\
& +\left|E_{(14,14)}\right|\left(14^{2}+14^{2}\right)+\left|E_{(14,16)}\right|\left(14^{2}+16^{2}\right)+\left|E_{(16,16)}\right|\left(16^{2}+16^{2}\right) \\
= & (2)\left(6^{2}+6^{2}\right)+(4)\left(6^{2}+12^{2}\right)+(4)\left(8^{2}+12^{2}\right)+(6 n-8)\left(8^{2}+14^{2}\right) \\
& (1)\left(12^{2}+12^{2}\right)+(6)\left(12^{2}+14^{2}\right)+(6 n-9)\left(14^{2}+14^{2}\right) \\
& +(6 n-12)\left(14^{2}+16^{2}\right)+\left(3 n^{2}-12 n+12\right)\left(16^{2}+16^{2}\right) \\
= & 1536 n^{2}+480 n-864 .
\end{aligned}
$$


Table 7. Edge partition of regular triangulene silicate networks $(\operatorname{RTSL}(n))$ with dimension $n$.

\begin{tabular}{|c|c|c|c|c|c|c|c|c|c|c|c|c|c|c|}
\hline$E_{(p, q)}$ & $E_{(12,12)}$ & $E_{(12,15)}$ & $E_{(12,24)}$ & $E_{(15,15)}$ & $E_{(15,24)}$ & $E_{(15,27)}$ & $E_{(18,24)}$ & $E_{(18,27)}$ & $E_{(18,30)}$ & $E_{(24,24)}$ & $E_{(24,27)}$ & $E_{(27,27)}$ & $E_{(27,30)}$ & $E_{(30,30)}$ \\
\hline$\left|E_{(p, q)}\right|$ & 2 & 4 & 4 & $3 n-2$ & 10 & $12 n-16$ & 2 & $6 n-8$ & $10 n-24$ & 1 & 6 & $6 n-7$ & $6 n-12$ & $3(n-2)^{2}$ \\
\hline
\end{tabular}

Table 8. Edge partition of regular triangulene oxide networks $(\operatorname{RTOX}(n))$ with dimension $n$.

\begin{tabular}{cccccccccc}
\hline$E_{(p, q)}$ & $E_{(6,6)}$ & $E_{(6,12)}$ & $E_{(8,12)}$ & $E_{(8,14)}$ & $E_{(12,12)}$ & $E_{(12,14)}$ & $E_{(14,14)}$ & $E_{(\mathbf{1 4}, \mathbf{1 6})}$ & $E_{(\mathbf{1 6}, \mathbf{1 6})}$ \\
\hline$\left|E_{(p, q)}\right|$ & 2 & 4 & 4 & $6 n-8$ & 1 & 6 & $6 n-9$ & $6 n-12$ & $3 n^{2}-12 n+12$ \\
\hline
\end{tabular}

Hence the theorem.

Theorem 9. The neighborhood version of the second Zagreb index $\left(M_{2}^{*}\right)$ of the regular triangulene silicate $(\operatorname{RTSL}(n))$ and oxide $(\operatorname{RTOX}(n)))$ networks for $n \geq 2$ is given by:

(i) $\quad M_{2}^{*}(\operatorname{RTSL}(n))=2700 n^{2}+12,285 n-16,713$,

(ii) $M_{2}^{*}(\operatorname{RTOX}(n))=768 n^{2}+120 n-380$.

Proof. The general formula of the neighborhood version of the second Zagreb index is given by:

$$
M_{2}^{*}(G)=\sum_{u v \in E(G)}\left[\delta_{G}(u) \delta_{G}(v)\right]
$$

(i) Let $G$ be the regular triangulene silicate network having dimension $n$. Applying the edge partition (6) and Table 7 on the definition of the neighborhood version of the second Zagreb index, we get the desired result as follows.

$$
\begin{aligned}
M_{2}^{*}(G)= & 2(12.12)+4(12.15)+4(12.24)+(3 n-2)(15.15)+10(15.24) \\
& +(12 n-16)(15.27)+2(18.24)+(6 n-8)(18.27)+(10 n \\
& -24)(18.30)+1(24.24)+6(24.27)+(6 n-7)(27.27) \\
& +(6 n-12)(27.30)+3(n-2)^{2}(30.30) \\
= & 2700 n^{2}+12,285 n-16,713 .
\end{aligned}
$$

(ii) Let $G$ be a regular triangulene oxide network having dimension $n$. Applying the edge partition (8) and Table 8, on the definition of the neighborhood version of the second Zagreb index, we get the desired result as follows.

$$
\begin{aligned}
M_{2}^{*}(G)= & 2(6.6)+4(6.12)+4(8.12)+2(3 n-4)(8.14)+1(12.12)+6(12.14) \\
& +3(2 n-3)(14.14)+6(n-2)(14.16)+3\left(n^{2}-4 n+4\right)(16.16) \\
= & 768 n^{2}+120 n-380 .
\end{aligned}
$$

Hence the proof.

Theorem 10. The neighborhood version of the hyper Zagreb index $\left(H M_{N}\right)$ of the regular triangulene silicate $(\operatorname{RTSL}(n))$ and oxide $(\operatorname{RTOX}(n)))$ networks for $n \geq 2$ is given by:

(i) $\quad H M_{N}(R T S L(n))=10,800 n^{2}+52,848 n-71,820$, 
(ii) $\quad \mathrm{HM}_{N}(\operatorname{RTOX}(n))=3072 n^{2}+720 n-1624$.

Proof. The general formula of the neighborhood version of the hyper Zagreb index is given by:

$$
H M_{N}(G)=\sum_{u v \in E(G)}\left[\delta_{G}(u)+\delta_{G}(v)\right]^{2}
$$

(i) Let $G \cong R T S L(n)$. Now, applying edge partition (6) and Table 7 on the definition of the $H M_{N}$ index, we get the following computation.

$$
\begin{aligned}
H M_{N}(G)= & 2(12+12)^{2}+4(12+15)^{2}+4(12+24)^{2}+(3 n-2)(15+15)^{2} \\
& +10(15+24)^{2}+(12 n-16)(15+27)^{2}+2\left((18+24)^{2}\right. \\
& +(6 n-8)(18+27)^{2}+(10 n-24)(18+30)^{2}+1(24+ \\
& 24)^{2}+6(24+27)^{2}+(6 n-7)(27+27)^{2}+(6 n- \\
& 12)(27+30)^{2}+3(n-2)^{2}(30+30)^{2} \\
= & 10,800 n^{2}+52,848 n-71,820 .
\end{aligned}
$$

(ii) Let $G \cong R T O X(n)$. Now, applying edge partition (8) and Table 8 on the definition of the $H M_{N}$ index, we get the following computation.

$$
\begin{aligned}
H M_{N}(G)= & 2(6+6)^{2}+4(6+12)^{2}+4(8+12)^{2}+(6 n-8)(8+14)^{2} \\
& +1(12+12)^{2}+6(12+14)^{2}+(6 n-12)(14+16)^{2} \\
& +\left(3 n^{2}-12 n+12\right)(16+16)^{2} \\
= & 3072 n^{2}+720 n-1624 .
\end{aligned}
$$

Hence the theorem.

Five topological indices for $\operatorname{RTSL}(n)$ and $\operatorname{RTOX}(n)$ are shown graphically in the Figure 9.

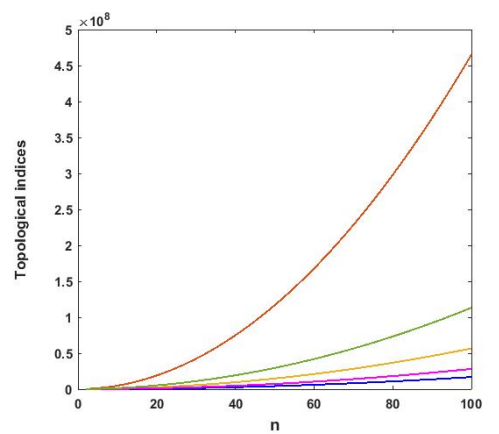

(a)

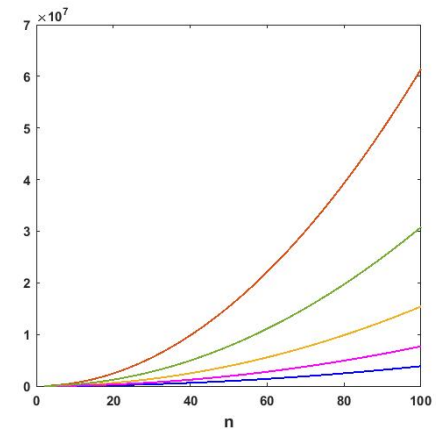

(b)

Figure 9. Different topological indices for regular triangulene (a) silicate and (b) oxide networks.

\subsection{Rhombus Silicate $(\operatorname{RHSL}(n))$ and Oxide $(\operatorname{RHOX}(n))$ Networks}

In this subsection, we obtain the five aforementioned indices for rhombus silicate $(R H S L(n))$ and oxide $(R H O X(n))$ networks. The structures of $R H S L(3)$ and $R H O X(3)$ are shown in Figures 10 and 11, respectively. For a rhombus silicate network $(R H S L(n))$ having dimension $n$, the number of nodes and 
edges is $5 n^{2}+2 n$ and $12 n^{2}$, respectively [21]. For a rhombus oxide network ( $\left.\operatorname{HOX}(n)\right)$ having dimension $n$, the number of nodes and edges is $3 n^{2}+2 n$ and $6 n^{2}$, respectively [21,26]. The vertex and edge partitions of the rhombus silicate network are as follows.

$$
\begin{gathered}
V(\operatorname{RHSL}(n))=V_{12} \cup V_{15} \cup V_{18} \cup V_{24} \cup V_{27} \cup V_{30}, \\
E(\operatorname{RHSL}(n))=E_{(12,12)} \cup E_{(12,24)} \cup E_{(15,24)} \cup E_{(18,24)} \cup E_{(24,27)} \cup E_{(15,15)} \cup E_{(18,27)} \\
\cup E_{(27,27)} \cup E_{(15,27)} \cup E_{(27,30)} \cup E_{(18,30)} \cup E_{(30,30)} .
\end{gathered}
$$

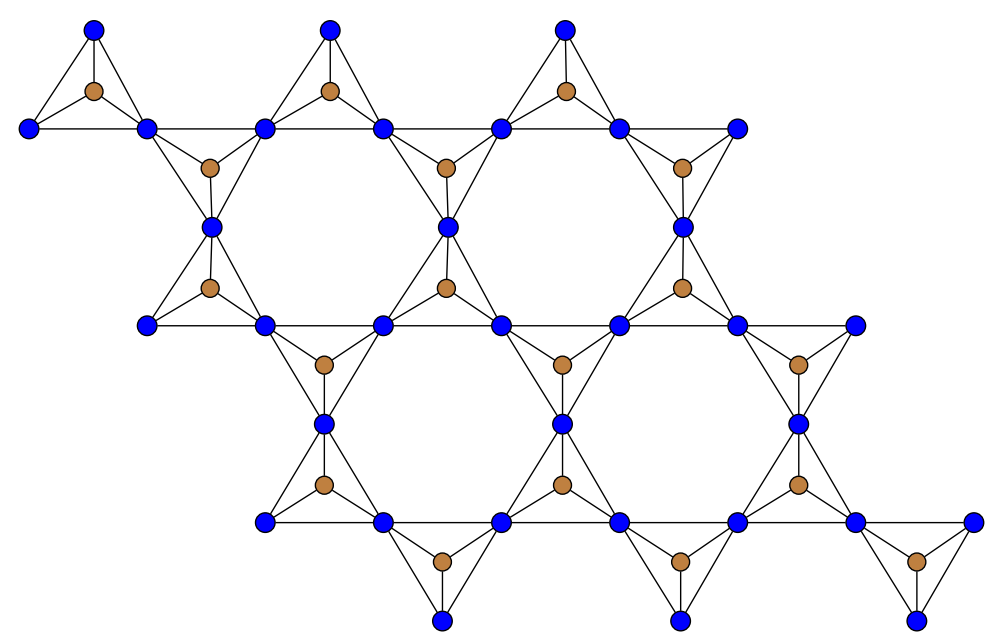

Figure 10. Rhombus-type silicate network (RHSL(3)).

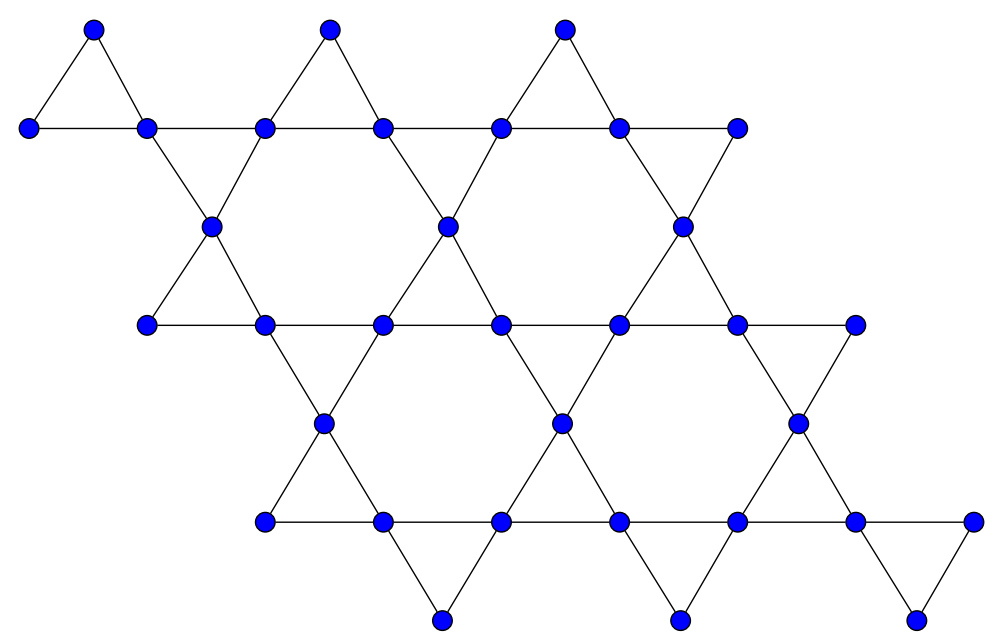

Figure 11. Rhombus-type oxide network (RHOX(3)).

The vertex and edge partitions of the rhombus oxide network are as follows.

$$
\begin{gathered}
V(R \operatorname{HOX}(n))=V_{6} \cup V_{8} \cup V_{12} \cup V_{14} \cup V_{16} \\
E(R \operatorname{HOX}(n))=E_{(6,6)} \cup E_{(6,12)} \cup E_{(8,12)} \cup E_{(8,14)} \cup E_{(12,14)} \cup E_{(14,14)} \cup E_{(14,16)} \cup E_{(16,16)} .
\end{gathered}
$$


Theorem 11. The neighborhood Zagreb index $\left(M_{N}\right)$ of the rhombus silicate $(R H S L(n))$ and oxide $\left.(R H O X(n))\right)$ networks for $n \geq 2$ is given by:

(i) $M_{N}(\operatorname{RHSL}(n))=1548 n^{2}+3636 n-4932$,

(ii) $\quad M_{N}(R H O X(n))=768 n^{2}-736 n+2048$.

Proof. (i) Let $G \cong R H S L(n)$. Now, applying vertex partition (9) and Table 9 on the definition of the neighborhood Zagreb index, we obtain:

$$
\begin{aligned}
M_{N}(G)= & \left|V_{12}\right|\left(12^{2}\right)+\left|V_{15}\right|\left(15^{2}\right)+\left|V_{18}\right|\left(18^{2}\right)+\left|V_{24}\right|\left(24^{2}\right)+\left|V_{27}\right|\left(27^{2}\right)+\left|V_{30}\right|\left(30^{2}\right) \\
= & 6\left(12^{2}\right)+8(n-1)\left(15^{2}\right)+2(n-1)^{2}\left(18^{2}\right)+(4)\left(24^{2}\right) \\
& +(8 n-12)\left(27^{2}\right)+(n-2)(n-1)\left(30^{2}\right) \\
= & 768 n^{2}-736 n+2048 .
\end{aligned}
$$

Table 9. Vertex partition of rhombus silicate networks $(R H S L(n))$ with dimension $n$.

\begin{tabular}{ccccccc}
\hline$V_{\boldsymbol{i}}$ & $\boldsymbol{V}_{\mathbf{1 2}}$ & $\boldsymbol{V}_{\mathbf{1 5}}$ & $\boldsymbol{V}_{\mathbf{1 8}}$ & $\boldsymbol{V}_{\mathbf{2 4}}$ & $\boldsymbol{V}_{\mathbf{2 7}}$ & $\boldsymbol{V}_{\mathbf{3 0}}$ \\
\hline$\left|V_{i}\right|$ & 6 & $8(n-1)$ & $2(n-1)^{2}$ & 4 & $8 n-12$ & $(n-2)(n-1)$ \\
\hline
\end{tabular}

(ii) Let $G \cong \operatorname{RHOX}(n)$. Now, using vertex partition (11) and Table 10 on the definition of the neighborhood Zagreb index, we obtain:

$$
\begin{aligned}
M_{N}(G)= & \left|V_{6}\right|\left(6^{2}\right)+\left|V_{8}\right|\left(8^{2}\right)+\left|V_{12}\right|\left(12^{2}\right)+\left|V_{14}\right|\left(14^{2}\right)+\left|V_{16}\right|\left(16^{2}\right) \\
= & 4\left(6^{2}\right)+4(n-1)\left(8^{2}\right)+4\left(12^{2}\right)+(8 n-12)\left(14^{2}\right) \\
& +\left(3 n^{2}-10 n+8\right)\left(16^{2}\right) .
\end{aligned}
$$

Table 10. Vertex partition of rhombus oxide networks $(R H O X(n))$ with dimension $n$.

\begin{tabular}{cccccc}
\hline$V_{\boldsymbol{i}}$ & $\boldsymbol{V}_{\mathbf{6}}$ & $V_{\mathbf{8}}$ & $V_{\mathbf{1 2}}$ & $V_{\mathbf{1 4}}$ & $V_{\mathbf{1 6}}$ \\
\hline$\left|V_{i}\right|$ & 4 & $4(n-1)$ & 4 & $8 n-12$ & $3 n^{2}-10 n+8$ \\
\hline
\end{tabular}

After simplifying and arranging the terms, the required result can be obtained easily. Hence the proof.

Theorem 12. The neighborhood version of the forgotten topological index $\left(F_{N}\right)$ of the rhombus silicate $(R H S L(n))$ and oxide $(\operatorname{RHOX}(n)))$ networks for $n \geq 2$ is given by:

(i) $F_{N}(R H S L(n))=38,664 n^{2}+80,136 n-131,868$,

(ii) $F_{N}(R H O X(n))=12,288 n^{2}-16,960 n+5568$. 
Proof. (i) Let $G \cong R H S L(n)$. Using vertex partition (9) and Table 9 on the above formula, we have:

$$
\begin{aligned}
F_{N}(G)= & 6\left(12^{3}\right)+8(n-1)\left(15^{3}\right)+2(n-1)^{2}\left(18^{3}\right)+(4)\left(24^{3}\right) \\
& +(8 n-12)\left(27^{3}\right)+(n-2)(n-1)\left(30^{3}\right) .
\end{aligned}
$$

After simplification and arranging the terms, the required result is obtained.

(ii) Let $G \cong R \operatorname{HOX}(n)$. Using vertex partition (11) and Table 10, the general formula of the $F_{N}$ index gives the following computation.

$$
\begin{aligned}
F_{N}(G)= & 4\left(6^{3}\right)+4(n-1)\left(8^{3}\right)+4\left(12^{3}\right)+(8 n-12)\left(14^{3}\right) \\
& +\left(3 n^{2}-10 n+8\right)\left(16^{3}\right) . \\
= & 768 n^{2}-736 n+2048 .
\end{aligned}
$$

Hence the proof.

Theorem 13. The modified neighborhood version of the forgotten topological index $\left(F_{N}^{*}\right)$ of the rhombus silicate $(\operatorname{RHSL}(n))$ and oxide $(\operatorname{RHOX}(n)))$ networks for $n \geq 2$ is given by:

(i) $F_{N}^{*}(\operatorname{RHSL}(n))=18,144 n^{2}-17,496 n+5472$,

(ii) $F_{N}^{*}(R H O X(n))=3072 n^{2}-3456 n+712$.

Proof. The general formula of the modified neighborhood version of the forgotten topological index is given by:

$$
F_{N}^{*}(G)=\sum_{u v \in E(G)}\left[\delta_{G}(u)^{2}+\delta_{G}(v)^{2}\right]
$$

(i) Let $G \cong \operatorname{RHSL}(n)$. Then, applying vertex partition (10) and Table 11 on the definition of the $F_{N}^{*}$ index, we have:

$$
\begin{aligned}
F_{N}^{*}(G)= & 6\left(12^{2}+12^{2}\right)+6\left(12^{2}+24^{2}\right)+8\left(15^{2}+24^{2}\right)+2\left(18^{2}+24^{2}\right) \\
& +8\left(24^{2}+27^{2}\right)+4(n-1)\left(15^{2}+15^{2}\right)+4(2 n-3)\left(18^{2}+27^{2}\right) \\
& +8(n-2)+2\left(27^{2}+27^{2}\right)+8(2 n-3)\left(15^{2}+27^{2}\right) \\
& +8(n-2)\left(27^{2}+30^{2}\right)+2(n-2)(3 n-4)\left(18^{2}+30^{2}\right) \\
& +6(n-2)^{2}\left(30^{2}+30^{2}\right) \\
= & 18,144 n^{2}-17,496 n+5472 .
\end{aligned}
$$

Table 11. Edge partition of rhombus silicate networks $(R H S L(n))$ with dimension $n$.

\begin{tabular}{ccccccccccccc}
\hline$E_{(p, q)}$ & $E_{(12,12)}$ & $E_{(12,24)}$ & $E_{(15,24)}$ & $E_{(15,27)}$ & $E_{(18,24)}$ & $E_{(18,27)}$ & $E_{(24,27)}$ & $E_{(15,15)}$ & $E_{(27,27)}$ & $E_{(27,30)}$ & $E_{(18,30)}$ & $E_{(30,30)}$ \\
\hline$\left|E_{(p, q)}\right|$ & 6 & 6 & 8 & $8(2 n-3)$ & 2 & $4(2 n-3)$ & 8 & $4(n-1)$ & $8(n-2)+2$ & $8(n-2)$ & $2(n-2)(3 n-4)$ & $6(n-2)^{2}$ \\
\hline
\end{tabular}


(ii) Let $G \cong R \operatorname{HOX}(n)$. Now, applying the vertex partition (12) and the Table 12 on the definition of the $F_{N}^{*}$ index, we have:

$$
\begin{aligned}
F_{N}^{*}(G)= & 2\left(6^{2}+6^{2}\right)+4\left(6^{2}+12^{2}\right)+4\left(8^{2}+12^{2}\right)+4(2 n-3)\left(8^{2}+14^{2}\right) \\
& +8\left(12^{2}+14^{2}\right)+2(4 n-7)\left(14^{2}+14^{2}\right)+8(n-2)\left(14^{2}+16^{2}\right) \\
& +6(n-2)^{2}\left(16^{2}+16^{2}\right) \\
= & 3072 n^{2}-3456 n+712 .
\end{aligned}
$$

Table 12. Edge partition of rhombus oxide networks $(\operatorname{RHOX}(n))$ with dimension $n$.

\begin{tabular}{ccccccccc}
\hline$E_{(p, q)}$ & $E_{(6,6)}$ & $E_{(6,12)}$ & $E_{(8,12)}$ & $E_{(8,14)}$ & $E_{(\mathbf{1 2}, 14)}$ & $E_{(14,14)}$ & $E_{(14,16)}$ & $E_{(16,16)}$ \\
\hline$\left|E_{(p, q)}\right|$ & 2 & 4 & 4 & $4(2 n-3)$ & 8 & $2(4 n-7)$ & $8(n-2)$ & $6(n-2)^{2}$ \\
\hline
\end{tabular}

Hence the theorem.

Theorem 14. The neighborhood versions of the second Zagreb index $\left(M_{2}^{*}\right)$ of the rhombus silicate $(R H S L(n))$ and oxide $(R H O X(n)))$ networks for $n \geq 2$ are given by:

(i) $M_{2}^{*}(\operatorname{RHSL}(n))=8640 n^{2}-8820 n+2142$,

(ii) $M_{2}^{*}(R H O X(n))=1536 n^{2}-1888 n-784$.

\section{Proof.}

(i) Let $G$ be the rhombus-type silicate network having dimension $n$. Applying the edge partition (10) and Table 11,on the definition of the neighborhood version of the second Zagreb index, we get the desired result as follows.

$$
\begin{aligned}
M_{2}^{*}(G)= & 6(12.12)+6(12.24)+8(15.24)+2(18.24)+8(24.27)+4(n-1)(15.15) \\
& +4(2 n-3)(18.27)+((n-2)+2)(27.27)+8(2 n-3)(15.27) \\
& +8(n-2)(27.30)+2(n-2)(3 n-4)(18.30)+6(n-2)^{2}(30.30) \\
= & 8640 n^{2}-8820 n+2142 .
\end{aligned}
$$

(ii) Let $G$ be the rhombus-type oxide network having dimension $n$. Applying the edge partition (12) and Table 12 on the definition of the neighborhood version of the second Zagreb index, we get the desired result as follows.

$$
\begin{aligned}
M_{2}^{*}(G)= & 2(6.6)+4(6.12)+4(8.12)+4(2 n-3)(8.14) \\
& +8(12.14)+2(4 n-7)(14.14)+8(n-2)(14.16)+6(n-2)^{2}(16.16) \\
= & 1536 n^{2}-1888 n-784 .
\end{aligned}
$$

Theorem 15. The neighborhood versions of the hyper Zagreb index $\left(H M_{N}\right)$ of the rhombus silicate $(R H S L(n))$ and oxide $(R H O X(n)))$ networks for $n \geq 2$ are given by:

(i) $\operatorname{HM}_{N}(\operatorname{RHSL}(n))=35,424 n^{2}-35,136 n+7956$,

(ii) $H M_{N}(R H O X(n))=6144 n^{2}-7232 n+1984$. 


\section{Proof.}

(i) Let $G \cong R H S L(n)$. Now, applying edge partition (10) and Table 11, on the definition of the $H M_{N}$ index, we get the following computation.

$$
\begin{aligned}
H M_{N}(G)= & 6(12+12)^{2}+6(12+24)^{2}+(4 n-4)(15+15)^{2}+8(15+24)^{2} \\
& +(16 n-24)(15+27)^{2}+2(18+24)^{2}+(8 n-12)(18+27)^{2} \\
& +2(n-2)(3 n-4)(18+30)^{2}+(8 n-14)(27+27)^{2} \\
& +(8 n-16)(27+30)^{2}+6(n-2)^{2}(30+30)^{2} \\
= & 35,424 n^{2}-35,136 n+7956 .
\end{aligned}
$$

(ii) Let $G \cong R H O X(n)$. Now, applying edge partition (12) and Table 12 on the definition of the $H M_{N}$ index, we get the following computation.

$$
\begin{aligned}
H M_{N}(G)= & 2(6+6)^{2}+4(6+12)^{2}+4(8+12)^{2}+(8 n-12)(8+14)^{2} \\
& +8(12+14)^{2}+(8 n-14)(14+14)^{2}+(8 n-16)(14+16)^{2} \\
& +6(n-2)^{2}(16+16)^{2} \\
= & 6144 n^{2}-7232 n+1984 .
\end{aligned}
$$

Hence the theorem.

The five topological indices for $\operatorname{RHSL}(n)$ and $\operatorname{RHOX}(n)$ are shown graphically in Figure 12.

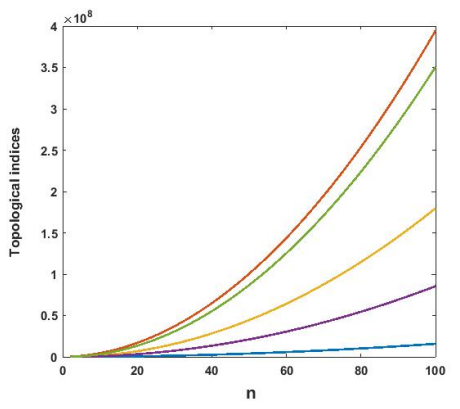

(a)

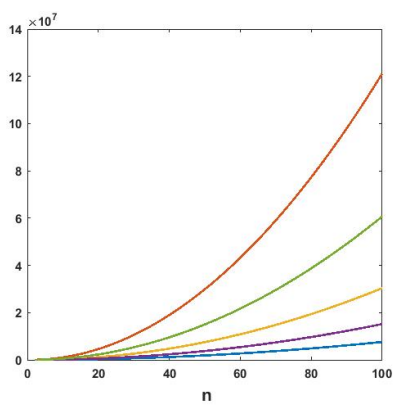

(b)

Figure 12. Different topological indices for rhombus-type (a) silicate and (b) oxide network.

\subsection{Copper (II) Oxide}

We obtain topological indices for copper (II) oxide $(\mathrm{CuO})$ here. Its molecular graph is depicted in Figure 13. In the $\mathrm{CuO}$ structure, the octagons are linked to each other in columns and rows. The connection between two octagons is achieved by creating one $C_{4}$ bond between two octagons. It contains $4 m n+3 n+m$ nodes and $6 m n+2 n$ edges, where $m$ and $n$ represent the number of octagons in rows and columns, respectively [22]. The vertex and edge partitions of the copper oxide network are as follows.

$$
\begin{gathered}
V(\mathrm{CuO})=V_{4} \cup V_{5} \cup V_{6} \cup V_{10} \cup V_{12}, \\
E(\mathrm{CuO})=E_{(4,4)} \cup E_{(4,5)} \cup E_{(4,6)} \cup E_{(5,6)} \cup E_{(6,6)} \cup E_{(6,10)} \cup E_{(10,10)} \cup E_{(10,12)} .
\end{gathered}
$$


Theorem 16. The neighborhood Zagreb index $\left(M_{N}\right)$ of copper oxide (CuO) for $m, n>2$ is given by:

$$
M_{N}(\mathrm{CuO})=380 m n-200 m-148 n+92 .
$$

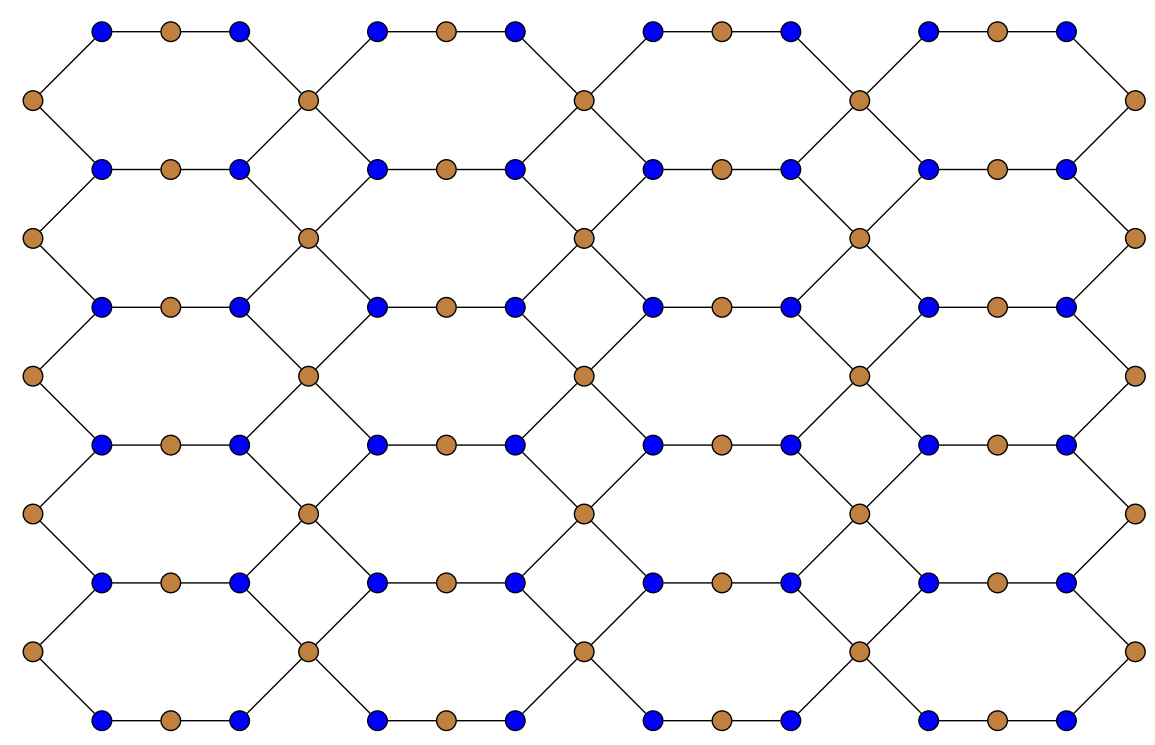

Figure 13. Copper oxide $(\mathrm{CuO})$ network with five rows and four columns.

Proof. The general formula of the neighborhood Zagreb index is given by:

$$
M_{N}(G)=\sum_{v \in V(G)} \delta_{G}(v)^{2}
$$

Let $G$ be a copper oxide network having $m$ rows and $n$ columns. Now, applying vertex partition (13) and Table 13 on the definition of the neighborhood Zagreb index, we get:

$$
\begin{aligned}
M_{N}(G)= & \left|V_{4}\right|\left(4^{2}\right)+\left|V_{5}\right|\left(5^{2}\right)+\left|V_{6}\right|\left(6^{2}\right)+\left|V_{10}\right|\left(10^{2}\right)+\left|V_{12}\right|\left(12^{2}\right) \\
= & (2 n+4)\left(4^{2}\right)+4\left(5^{2}\right)+(m n+4 m+3 n-10)\left(6^{2}\right)+(2 m n-2 m)\left(10^{2}\right) \\
& +(m n-m-2 n+2)\left(12^{2}\right) \\
= & 380 m n-200 m-148 n+92 .
\end{aligned}
$$

Table 13. Vertex partition of copper oxide $(\mathrm{CuO})$ with $m$ rows and $n$ columns.

\begin{tabular}{cccccc}
\hline$V_{\boldsymbol{i}}$ & $\boldsymbol{V}_{\mathbf{4}}$ & $\boldsymbol{V}_{\mathbf{5}}$ & $\boldsymbol{V}_{\mathbf{6}}$ & $\boldsymbol{V}_{\mathbf{1 0}}$ & $\boldsymbol{V}_{\mathbf{1 2}}$ \\
\hline$\left|V_{i}\right|$ & $2 n+4$ & 4 & $m n+4 m+3 n-10$ & $2 m n-2 m$ & $m n-m-2 n+2$ \\
\hline
\end{tabular}

Hence the proof.

Theorem 17. The neighborhood version of the forgotten topological index $\left(F_{N}\right)$ of copper oxide $(\mathrm{CuO})$ for $m, n>2$ is given by:

$$
F_{N}(C u O)=3944 m n-2864 m-2680 n+2052 .
$$


Proof. Applying vertex partition (13) and Table 13 on the definition of the neighborhood version of the forgotten topological index, the required result can be obtained easily like the previous proof.

Theorem 18. The modified neighborhood version of the forgotten topological index $\left(F_{N}^{*}\right)$ of copper oxide (CuO) for $m, n>2$ is given by:

$$
F_{N}^{*}(\mathrm{CuO})=1248 m n-816 m-672 n+508 .
$$

Proof. Let $G$ be a copper oxide network with $m$ rows and $n$ columns. Now, by the edge partition (14) and Table 14, we have from the general formulation of $F_{N}^{*}$ the following.

$$
\begin{aligned}
F_{N}^{*}(G)= & \left|E_{(4,4)}\right|\left(4^{2}+4^{2}\right)+\left|E_{(4,5)}\right|\left(4^{2}+5^{2}\right)+\left|E_{(4,6)}\right|\left(4^{2}+6^{2}\right)+\left|E_{(5,6)}\right|\left(5^{2}\right. \\
& \left.+6^{2}\right)+\left|E_{(6,6)}\right|\left(6^{2}+6^{2}\right)+\left|E_{(6,10)}\right|\left(6^{2}+10^{2}\right) \\
& +\left|E_{(10,10)}\right|\left(10^{2}+10^{2}\right)+\left|E_{(10,12)}\right|\left(10^{2}+12^{2}\right) \\
= & (4)\left(4^{2}+4^{2}\right)+(4)\left(4^{2}+5^{2}\right)+(4 n-4)\left(4^{2}+6^{2}\right)+(4)\left(5^{2}+6^{2}\right) \\
& +(6 m-10)\left(6^{2}+6^{2}\right)+(2 m n-2 m+2 n-2)\left(6^{2}+10^{2}\right) \\
& +(4 n-4)\left(10^{2}+10^{2}\right)+(4 m n-4 m-8 n+8)\left(10^{2}+12^{2}\right) .
\end{aligned}
$$

Table 14. Edge partition of copper oxide $(\mathrm{CuO})$ with $m$ rows and $n$ columns.

\begin{tabular}{ccccccccc}
\hline$E_{(p, q)}$ & $E_{(\mathbf{4}, \mathbf{4})}$ & $E_{(4,5)}$ & $E_{(\mathbf{4 , 6})}$ & $E_{(5,6)}$ & $E_{(\mathbf{6}, \mathbf{6})}$ & $E_{(\mathbf{6 , 1 0})}$ & $E_{(\mathbf{1 0}, \mathbf{1 0})}$ & $E_{(\mathbf{1 0 , 1 2})}$ \\
\hline$\left|E_{(p, q)}\right|$ & 4 & 4 & $4 n-4$ & 4 & $6 m-10$ & $2 m n-2 m+2 n-2$ & $4 n-4$ & $4 m n-4 m-8 n+8$ \\
\hline
\end{tabular}

The required result is obvious after simplification.

Theorem 19. The neighborhood version of the second Zagreb index $\left(M_{2}^{*}\right)$ of copper oxide $(\mathrm{CuO})$ for $m, n>2$ is given by:

$$
M_{2}^{*}(C u O)=600 m n-384 m-344 n+248 .
$$

Proof. Applying edge partition (14) and Table 14 on the the general formula of the $M_{2}^{*}$ index, the required result can be obtained easily like the previous proof.

Theorem 20. The neighborhood version of the hyper Zagreb index $\left(H M_{N}\right)$ of copper oxide $(\mathrm{CuO})$ for $m, n>2$ is given by:

$$
H M_{N}(C u O)=2448 m n-1584 m-1360 n+984 .
$$

Proof. Applying edge partition (14) and Table 14 on the the general formula of the $M_{2}^{*}$ index, the required result follows in the same way as before.

The five topological indices for $\mathrm{CuO}$ are depicted graphically in Figures 14-16. 


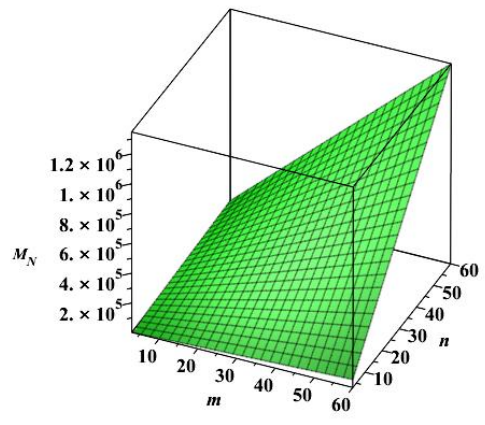

(a)

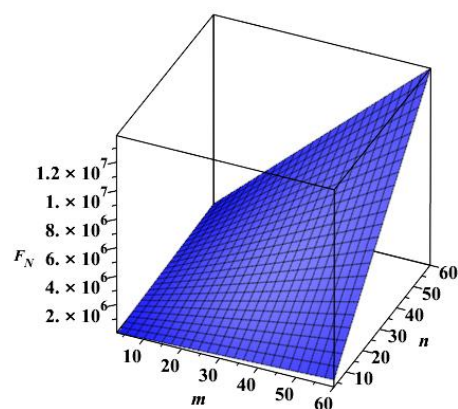

(b)

Figure 14. (a) The $M_{N}$ index and (b) the $F_{N}$ index of the copper oxide network.

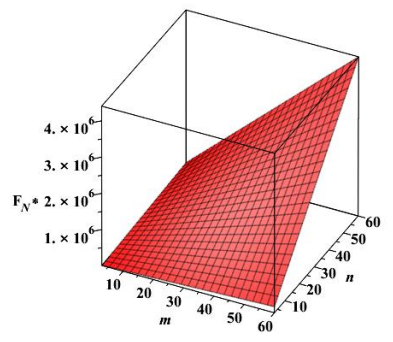

(a)

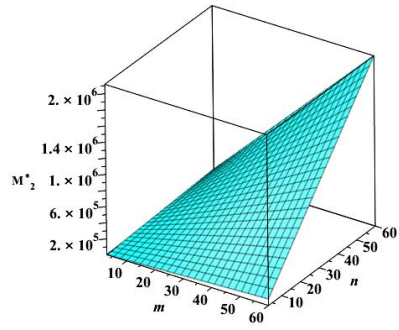

(b)

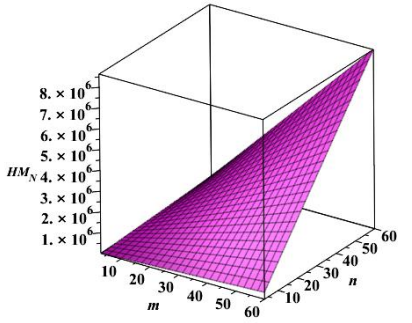

(c)

Figure 15. (a) The $F_{N}^{*}$ index, (b) the $M_{2}^{*}$ index, and (c) the $H M_{N}$ index of the copper oxide network.

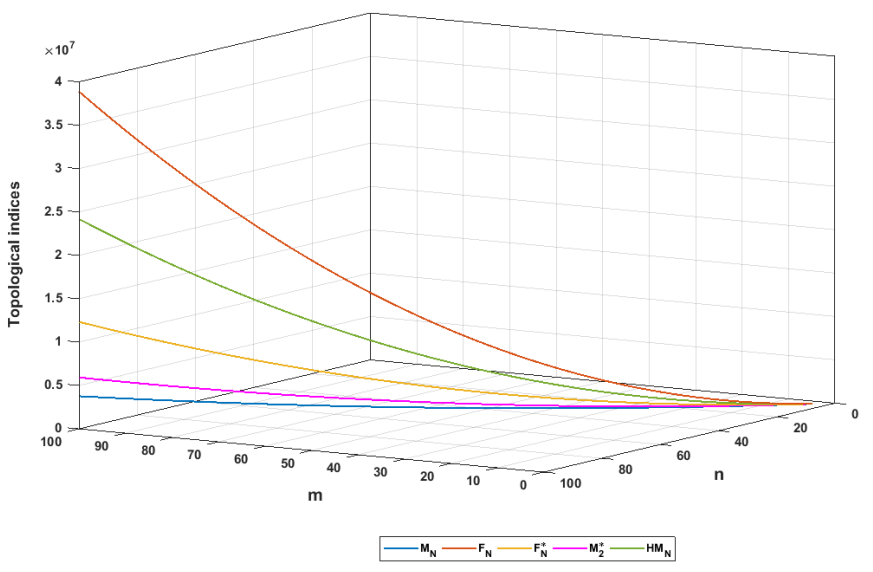

Figure 16. Different topological indices for the copper oxide network.

\section{Remarks and Conclusions}

In this work, we derived some explicit expressions of five topological indices, namely the neighborhood Zagreb index $\left(M_{N}\right)$, the neighborhood version of the forgotten topological index $\left(F_{N}\right)$, the modified neighborhood version of the forgotten topological index $\left(F_{N}^{*}\right)$, the neighborhood version of the second Zagreb index $\left(M_{2}^{*}\right)$, and the neighborhood version of the hyper Zagreb index $\left(H M_{N}\right)$, for dominating silicate $(D S L(n))$ and oxide $(\operatorname{DOX}(n))$ networks, regular triangulene silicate $(R T S L(n))$ and oxide $(\operatorname{RTOX}(n))$ networks, rhombus-shaped silicate $(R H S L(n))$ and oxide $(R H O X(n))$ networks, and the copper oxide $(\mathrm{CuO})$ network. Furthermore, we made a comparison graphically among all indices for the above-mentioned networks. The comparison was made in Figures 6, 9, 12, and 16. The aforementioned 
indices were very close at the initial stage of the given domain and then grew. Among the five topological indices, the neighborhood version of the forgotten topological index $\left(F_{N}\right)$ had the most dominating power in comparison with the other indices. The neighborhood Zagreb index $\left(M_{N}\right)$ grew more slowly than the other indices. The nature of the neighborhood version of the second Zagreb index $\left(M_{2}^{*}\right)$ was close to the $F_{N}$ index. In comparison to other networks, the $H M_{N}$ index was close to the $F_{N}$ index in $R T S L(n)$. In $\operatorname{RTSL}(n)$, the gap between the $F_{n}$ and $H M_{N}$ indices was more than the other networks. From the vertical axis, it was clear that the indices for different networks grew in the following order:

$$
C u O<\operatorname{RTOX}(n)<\operatorname{RHOX}(n)<\operatorname{RHSL}(n)<\operatorname{RTSL}(n)<\operatorname{DOX}(n)<\operatorname{DSL}(n),
$$

where in each case, the indices had the following order:

$$
M_{N}<M_{2}^{*}<F_{N}^{*}<H M_{N}<F_{N}
$$

i.e., all the indices behaved differently in each network discussed above. This work will help researchers working in network science understand the topology of the aforementioned networks. In the future, we would like to obtain these indices for some other chemical networks, trees, dendrimers, etc.

Author Contributions: Conceptualization, N.D.; Methodology, N.D., S.M.; Writing-Original Draft Preparation, N.D., S.M. and A.P.; Writing-Review and Editing, N.D., S.M. and A.P.; Supervision, N.D., A.P.; Project Administration, A.P.

Funding: This research was funded by the Department of Science and Technology (DST), the Government of India (Inspire Fellowship [IF170148]).

Acknowledgments: We acknowledge the anonymous reviewers for their important comments.

Conflicts of Interest: The authors declare no conflict of interest.

\section{References}

1. Borkow, G. Safety of Using Copper Oxide in Medical Devices and Consumer Products. Cur. Chem. Biol. 2012, 6, 86-92.

2. Trinajstić, N. Chemical Graph Theory; CRC Press: Boca Raton, FL, USA, 1983.

3. Gutman, I.; Polansky, O.E. Mathematical Concepts in Organic Chemistry; Springer: Berlin, Germany, 1986.

4. Diudea, M.V.; Gutman, I.; Lorentz, J. Molecular Topology; Babes-Bolyai University: Cluj-Napoca, Romania, 2001.

5. Wiener, H. Structural determination of the paraffin boiling points. J. Am. Chem. Soc. 1947, 69, 17-20. [CrossRef] [PubMed]

6. Gutman, I.; Trinajstić, N. Graph theory and molecular orbitals.Total $\pi$-electron energy of alternate hydrocarbons. Chem. Phys. Lett. 1972, 17, 535-538. [CrossRef]

7. Furtula, B.; Gutman, I. Forgotten topological index. J. Math. Chem. 2015, 53. [CrossRef]

8. Shirdel, G.H.; Rezapour, H.; Sayadi, A.M. The hyper Zagreb index of graph operations. Iran. J. Math. Chem. 2013, 4, 213-220.

9. Mondal, S.; De, N.; Pal, A. The Neighborhood Zagreb index of product graphs and its chemical interest. arXiv 2018, arXiv:1805.05273.

10. Mondal, S.; De, N.; Pal, A. On some new neighborhood degree based indices. Acta Chem. IASI 2019, Accepted.

11. Aslam, A.; Bashir, Y.; Ahmed, S.; Gao, W. On topological indices of certain dendrimer structures. Z. Naturforsch. 2017, 72, 559-566. [CrossRef]

12. Imran, M.; Siddiqui, M.K.; Abunamous, A.A.E.; Adi, D.S.; Rafique, H.; Baig, A.Q. Eccentricity Based Topological Indices of an Oxide Network. Mathematics 2018, 6, 126. [CrossRef]

13. De, N. On Molecular Topological Properties of $\mathrm{TiO}_{2}$ Nanotubes. J. Nanosci. 2016. [CrossRef]

14. Bashir, Y.; Aslam, A.; Kamran, M.; Qureshi, M.I.; Jahangir, A.; Rafiq, M.; Bibi, N.; Muhammad, N. On Forgotten Topological Indices of Some Dendrimers Structure. Molecules 2017, 22, 867. [CrossRef] 
15. Akhter, S.; Imran, M.; Gao, W.; Farahani, M.R. On topological indices of honeycomb networks and graphene networks. Hacet. J. Math. Stat. 2018, 47, 19-35. [CrossRef]

16. Kwun, Y.C.; Ahmad, J.; Nazeer, W.; Khalid, W.; Kang, S.M. Topological invariants of molecular graph of Graphene. JP J. Geom. Topol. 2018, 21, 95-103. [CrossRef]

17. Jagadeesh, R.; Khanna, M.R.R.; Indumathi, R.S. Some results on topological indices of graphene. Nanomater. Nanotechnol. 2016, 6, 1-6.

18. Akhtar, S.; Imran, M. On molecular topological properties of benzenoid structures. Can. J. Chem. 2016, 94, 687-698. [CrossRef]

19. Shegehalli, V.S.; Kanabur, R. Computation of New Degree-Based Topological Indices of Graphene. J. Math. 2016, 2016, 4341919. [CrossRef]

20. Baig, A.Q.; Imran, M.; Ali, H. On topological indices of poly oxide, poly silicate, DOX, and DSL networks. Can. J. Chem. 2015, 93, 730-739. [CrossRef]

21. Javaid, M.; Rehman, M.U.; Cao, J. Topological indices of rhombus type silicate and oxide networks. Can. J. Chem. 2017, 95, 134-143. [CrossRef]

22. Gao, W.; Baig, A.Q.; Khalid, W.; Farahani, M.R. Molecular description of copper(II) oxide. Maced. J. Chem. Chem. Eng. 2017, 36, 93-99.

23. Gao, W.; Siddiqui, M.K. Molecular Descriptors of Nanotube, Oxide, Silicate, and Triangulene Networks. J. Chem. 2017. [CrossRef]

24. Rajan, B.; William, A.; Grigorious, C.; Stephen, S. On Certain Topological Indices of Silicate, Honeycomb and Hexagonal Networks. J. Comput. Math. Sci. 2012, 3, 530-535.

25. Ghorbani, M.; Hosseinzadeh, M.A. The third version of Zagreb index. Discret. Math. Algorithms Appl. $2013,5$. [CrossRef]

26. Simonraj, F; George, A. Topological Properties of few Poly Oxide, Poly Silicate, DOX and DSL Networks. Int. J. Future Comput. Commun. 2013, 2. [CrossRef]

(C) 2019 by the authors. Licensee MDPI, Basel, Switzerland. This article is an open access article distributed under the terms and conditions of the Creative Commons Attribution (CC BY) license (http:/ / creativecommons.org/licenses/by/4.0/). 